\title{
Open-Access Silicon Photonics: Current Status and Emerging Initiatives
}

\author{
Abdul Rahim, Thijs Spuesens, Roel Baets, Fellow, IEEE, Wim Bogaerts SM, IEEE
}

\begin{abstract}
Silicon Photonics is widely acknowledged as a gamechanging technology, driven by the needs of datacom and telecom. Silicon Photonics builds on highly capital-intensive manufacturing infrastructure, and mature open-access silicon photonics platforms are translating the technology from research fabs to industrial manufacturing levels. To meet the current market demands for silicon photonics manufacturing, a variety of openaccess platforms is offered by CMOS pilot lines, $R \& D$ institutes and commercial foundries. This paper presents an overview of existing and upcoming commercial and non-commercial openaccess silicon photonics technology platforms. We also discuss the diversity in these open-access platforms and their key differentiators.
\end{abstract}

Index Terms-CMOS, foundry, multi-project wafer (MPW), open-access, photonic integrated circuits, photonic manufacturing, silicon photonics.

\section{INTRODUCTION}

$\mathbf{S}$ ILICON Photonics is a technology that implements highdensity photonic integrated circuits (PIC) with complex functionality using process technology of a CMOS electronics fab. Leveraging the existing CMOS infrastructure makes silicon photonics very well positioned to fabricate low cost, high yield, small form-factor and low power PICs, and at the same time scale to large commercial volumes [1]-[3]. The above definition of silicon photonics holds for a variety of material systems. To name a few, this includes different types of Silicon-on-Insulator (SOI), Silicon Nitride-on-Insulator (SiN), Germanium-onInsulator (GOI), Germanium-on-Silicon, Germanium-on-SOI, Germanium-on-Silicon Nitride and Silicon-on-Silicon Nitrideon-Silicon. Since all these material systems can use wellestablished CMOS processing methodologies and infrastructure for densely integrated PICs, they can be considered as different flavors of silicon photonics. But to date the SOI platform is the most prominent of these material systems both in terms of technological maturity and commercial use.

The last decade has seen the transformation of silicon photonics from a promising research field to a commercial success, mainly driven by the needs for large volumes of high-speed links for datacenters, metro communication and telecommunication. However, the first conceived applications of silicon photonics were for sensing applications such as fiber gyroscopes [4]. Nevertheless, variable optical attenuators

Abdul Rahim, Thijs Spuesens, Roel Baets and Wim Bogaerts are with the Photonics Research Group, Department of Information Technology, Ghent University - imec and Center for Nano- and Biophotonics, Ghent University, Ghent B-9000, Belgium (e-mail: abdul.rahim@ugent.be, thijs.spuesens@ugent.be, roel.baets@ugent.be,wim.bogaerts@ugent.be). Roel Baets and Abdul Rahim coordinate ePIXfab, the European Silicon Photonics alliance.

Manuscript received May 22, 2018; revised August xx, 20xx.
(VOA) for passive optical networks (PON), developed and commercialized by Bookham Technologies in 1997 (with the technology later taken up by Kotura and afterwards by Mellanox) were one of the first silicon photonics products to be actually commercialized [4]. Building up to the dotcom boom (and subsequent bust) in the early 2000s, silicon photonics was being considered as a technology that could address the demands of emerging tele/data communication applications. The bursting of the dot-com bubble did little to reduce that expectation, but had an obvious negative effect on the investment climate for silicon photonics. It is only in 2007 that we see transceivers for datacenter communication, developed by Luxtera, to appear on the market and become a volume-manufactured silicon photonics product [5], [6] In 2012 Cisco, which is one of the biggest networking companies, acquired silicon photonics company Lightwire and started introducing silicon photonics solutions in their products, such as CPAK $^{\circledR}$ 100GBASE fiber modules. And in 2014, Acacia Communications brought silicon photonics into the commercial telecommunication market, launching coherent silicon photonics modules for intra-data center communication and long-haul DWDM links. In 2016, chip manufacturer Intel, which has been building its silicon photonics technology for over a decade [7], [8], launched a $100 \mathrm{~Gb} / \mathrm{s}$ parallel singlemode fiber 4-lane (PSM4) transceiver for cloud and data center applications. Inphi announced in 2017 to offer inter-datacenter 100G PAM4 DWDM silicon photonics optical engine. Figure 1 summarizes some of the major technical and commercial milestones achieved in the field of silicon photonics in the last 30+ years. The milestones mentioned above and in Fig. 1 are just a few examples of the many milestones accomplished by the field of silicon photonics, too numerous to report here completely.

Over the years there has been a manifold increase in the number of fabless silicon photonics chip and system vendors serving the data center transceiver market. Consequently, in 2017 the silicon photonics transceiver market growth has outpaced the total optical transceiver market by a significant margin [9], [10]. Furthermore, the traction of the technology platforms is stimulating a diversification in the application space: extensive R\&D initiatives and start-up companies worldwide are betting on silicon photonics for a wide range of applications such as sensing and bio-photonics [11]-[13], LiDAR for automotive industry [14], [15], neuromorphic computing [16]-[18], machine learning [19], [20], quantum information processing [21], [22] and many more.

Figure 2 is showing a simplified representation of the evolution silicon photonics manufacturing has gone through 


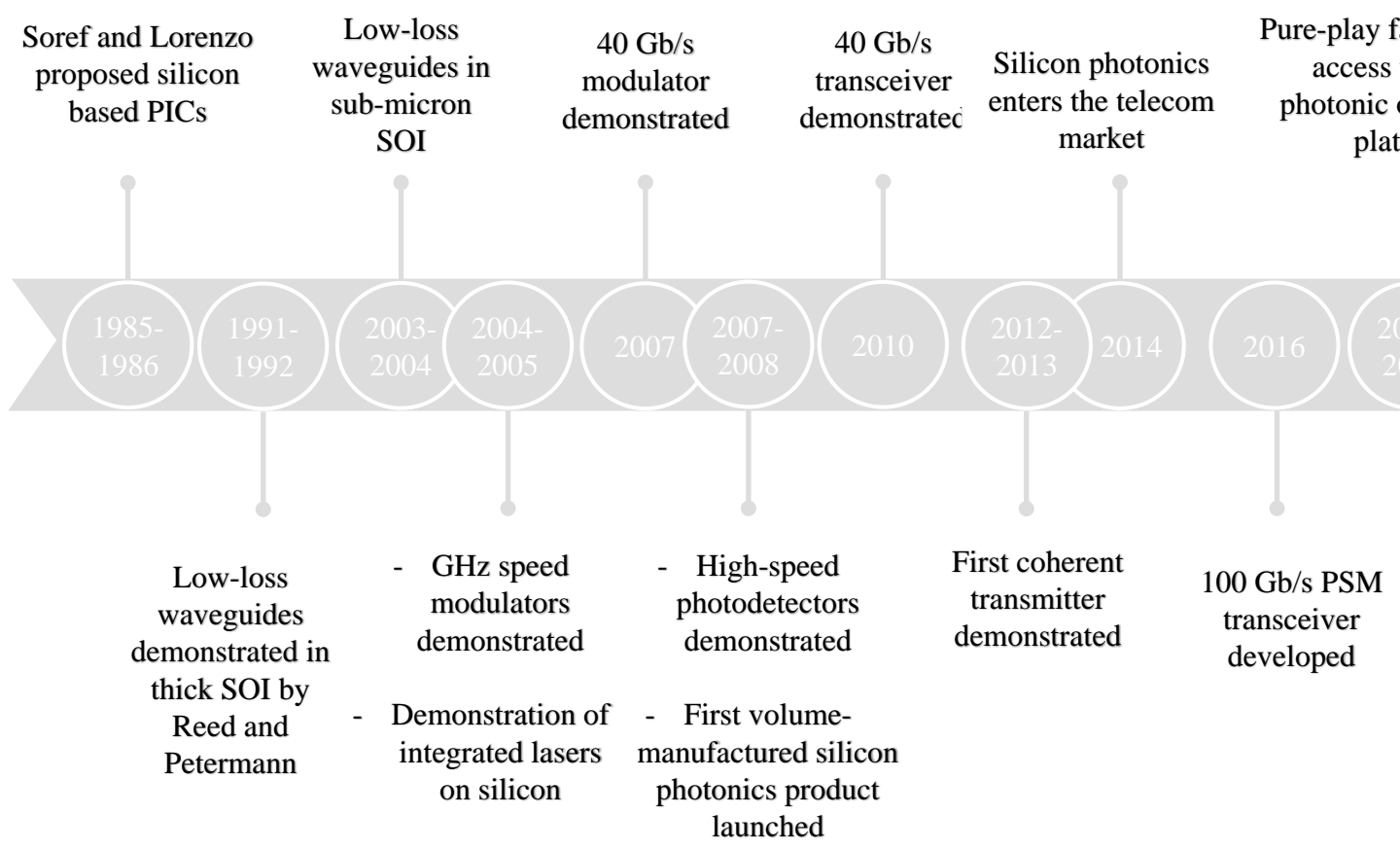

Fig. 1: Timeline of some of the major milestones in the field of silicon photonics.

in the last several years. The manufacturing process is crucial to the success of silicon photonics, but the capital investment cost for CMOS fabrication infrastructure sets a limit to the number of actors who can fabricate such devices. Similar to the ecosystem of CMOS electronics, we can identify three types of manufacturers: integrated device manufacturers (IDM) are vertically integrated and have their own infrastructure to manufacture silicon photonics chips for their own products. In stark contrast, pure-play foundries have an open-access manufacturing process, where the fabs offer PIC manufacturing services that can be used hands-off by third party external users/clients such as fabless companies. The notion "openaccess" does not mean that the manufacturing services are free of cost to the end-users, but rather that the fab imposes little or no restrictions to fabricate PICs for third parties. This is contrary to other areas of commerce where open-access implies that there is no cost to the end-users (for example, open-access publications). Anticipating a growing demand for silicon photonics PIC manufacturing, such pure-play foundries (Advanced Micro Foundry, GlobalFoundries, TowerJazz and others) have emerged in recent years to offer open-access silicon photonics technologies [23] to cater various manufacturing volumes. The third type of manufacturer is the CMOS pilot line at an advanced (public or private) research institute [24]-[32]. They offer open-access to their platforms, which includes multiproject-wafer (MPW) fabrication, and this has allowed these institutes to continuously improve their technology offering, to a point where the high technology readiness level (TRL) of their platforms has enabled them to offer low-volume manufacturing of silicon photonic chips through bilateral contracts. In some cases the R\&D institute/pilot line uses the same process flow as a commercial fab to ease the transfer of manufacturing process from R\&D institutes to commercial fabs. Consequently, an entire ecosystem of companies providing PIC design support, equipment, and services for packaging, testing and assembly of PICs is very rapidly materializing, supporting fabless companies engaged in silicon photonics product development [33]-[35].

This paper gives an overview of the current status of the openaccess silicon photonics technologies and their access model. We draw parallels with the CMOS electronics ecosystem which has similar models, but for much larger manufacturing volumes. The diversity of open-access silicon photonics technologies and its impact on economy-of-scale is discussed. Recent improvements in the design methodologies for PIC design and initiatives towards high-volume manufacturing are also discussed. It is to be understood that silicon photonics is a rapidly evolving technology in a continuously changing economic landscape, implying that the specific details about the current manufacturing platforms will likely be outdated soon. However, the trends which we discuss in this paper are likely to hold for the foreseeable future. It is important to mention that the discussion presented in this paper is based solely on publicly accessible information, not only peer-reviewed articles but also information about the open-access platforms publicized by their providers.

\section{ECONOMY OF (WAFER) SCALE FOR A STANDARDIZED PLATFORM}

A standardized silicon photonics platform (also termed a "generic integration platform" [36]) is one for which the manufacturing volumes are sufficiently large to establish and maintain a mature process flow. Such a platform has to offer a set of basic passive and active photonic functions, making it suitable for different applications and even for future performance scaling. This is required to offer the standardized technology as an open-access platform in a fabless model, 


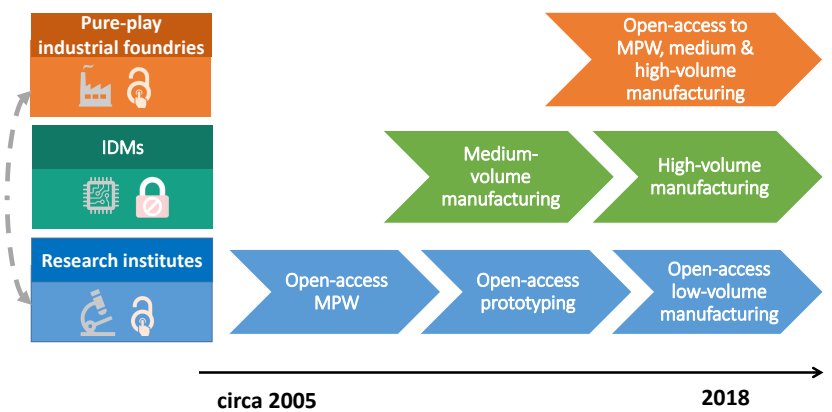

Fig. 2: Simplified representation of the evolution of silicon photonics open-access platforms provided by the research institutes and the pure-play fabs for different volumes of manufacturing. The evolution of the proprietary platforms developed by integrated device manufacturers (IDM) is also represented. It must be noted that in some cases the IDMs also provide access to their technology platforms through bilateral contracts with third-parties. The grey dashed line represents the collaboration to transfer the manufacturing process from $R \& D$ institutes to commercial pure-play fabs.

where the end-user has to adhere strictly to the process and design rules defined by the fab. These rules typically prohibit the end-user to change key platform features such as the material stack, critical dimensions, number of etch levels, doping concentrations, etc.

Leveraging existing and well-established CMOS manufacturing technology and infrastructure has catapulted silicon photonics to the forefront of photonic integration technologies. However, the manufacturing volumes needed to meet even today's largest volume applications for PICs are meager (by several orders of magnitude) when compared to the manufacturing volumes of CMOS electronics. A typical CMOS fab processes up to tens of thousands of wafers per month. In contrast, the current total silicon photonics market requires up to few tens of thousands of wafers per year [37]. Considering this disparity, it makes perfect economic sense to capitalize on existing CMOS fabs with established processes, rather than making excessive capital investments for building dedicated fab infrastructure for silicon photonics. Apart from the technological differentiators, this is one fundamental economic reason that distinguishes silicon photonics from competing PIC technologies based on III-V semiconductor, dielectrics, polymers, etc.

Figure 3 shows the approximate chip cost $/ \mathrm{mm}^{2}$ for a standardized silicon photonics technology in a CMOS fab, which is close to being fully loaded with CMOS electronics manufacturing. The cost curve is based on inputs provided by various fabs providing prototyping and/or manufacturing services for silicon photonics PICs. Here the term prototyping refers to volumes of less than $1 \mathrm{~K}$ chips whereas volumes of less than $100 \mathrm{~K}$ chips are classified as medium volume. It is important to highlight that the volume definitions mentioned above are valid only for PIC manufacturing as any existing volume manufacturing of PICs lies well within the low volume bracket of CMOS electronics manufacturing. The cost curve assumes a chip area of $25 \mathrm{~mm}^{2}$. It accounts for the

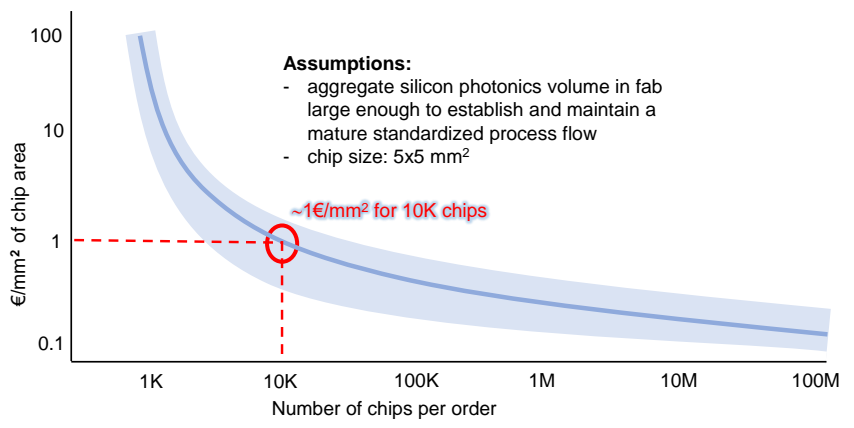

Fig. 3: Chip cost per $\mathrm{mm}^{2}$ for silicon photonics chips in a CMOS fab, shared with non-photonics use. The cost curve assumes that the silicon photonics volume in the fab is large enough to establish and maintain a mature process flow offering passive and active building blocks. Data collected from members of ePIXfab - The European Silicon Photonics Alliance (http://epixfab.eu).

infrastructure for a complete silicon photonics process flow including specific capital expenditures to set up the dedicated process for germanium growth needed for the fabrication of photodetectors in a CMOS fab.

The cost $/ \mathrm{mm}^{2}$ of chip area in Fig. 3 is estimated for standardized platforms supporting either monolithic or hybrid integration with electronics. Moreover, the chips considered comprise both active and passive photonic building blocks. The cost curve shows that for $10 \mathrm{~K}$ chips, each $\mathrm{mm}^{2}$ of chip area costs only $1 €$ (or $1 \$$, given that these numbers are based on order-of-magnitude calculations). This contradicts directly the gross misunderstanding that silicon photonics is viable only for very large volumes. For up to $1 \mathrm{~K}$ chips, the cost is calculated by considering MPW fabrication, which is a cost-effective mechanism for the prototyping of a small number of PICs [38]. The fabrication cost of $10 \mathrm{~K}$ chips is calculated by considering a full lot comprising $25200 \mathrm{~mm}$ wafers (The number of chips on $300 \mathrm{~mm}$ wafers is roughly double, so still within the same order of magnitude). It must be noted that the cost of packaging is not considered in Fig. 3. Furthermore, it is assumed that the fabrication has a high yield due to the high maturity of the technology platform.

\section{DIFFERENT FORMS OF OPEN-ACCESS SILICON PHOTONICS PLATFORMS}

As mentioned in section I there is a variety of material systems that fulfills the definition of silicon photonics, which is the ability to use existing CMOS infrastructure for the implementation of complex photonic functions and systems on a chip. In the next sub-sections, a brief description is provided for today's most prominent flavors of silicon photonics. This section only discusses those flavors of silicon photonics that are already or will be available in an open-access mode. In the current landscape, most material systems are developed for optical communication applications in C- and O-band, with wavelengths between $1300 \mathrm{~nm}$ and $1600 \mathrm{~nm}$. Platforms for visible and mid-IR wavelengths are also developed on some of the material systems mentioned below but those developments 
are still marginal as compared to the developments made for the telecommunication wavelength range.

\section{A. Silicon-on-Insulator (SOI)}

Silicon-on-Insulator is the most mature silicon photonics material platform. It relies on silicon (with a high refractive index) as the waveguide core material, surrounded on all sides by a silicon dioxide (glass) cladding. This gives a $41 \%$ index contrast, which is defined as $\left(n_{\text {core }}^{2}-n_{\text {clad }}^{2}\right) /\left(2 \times n_{\text {core }}^{2}\right)$, in the telecommunication window. SOI can again be subdivided in two classes, namely sub-micron SOI and and thick SOI. The classification of sub-micron SOI and thick SOI is based on the thickness of the silicon guiding layer (i-e, the device layer of photonic components) and not the thickness of the buried thermal oxide (BOX). For thick SOI the guiding layer is typically larger than $1 \mu \mathrm{m}$ whereas for sub-micron SOI the guiding layer thickness is well below $1 \mu \mathrm{m}$. For both classes of SOI used in silicon photonics, the BOX layer is usually thick enough to prevent the leakage of optical signal into the substrate. Another distinction the two have is their current wafer size. Unlike thick SOI, sub-micron SOI is available in wafer size of $200 \mathrm{~mm}$ ( 8 inch) or more.

1) Sub-micron SOI: In terms of economic activity, wafer volume and number of actors in the fabrication field, submicron SOI is by far the dominant flavor of silicon photonics, and is often considered as a synonym for the entire field. Openaccess sub-micron SOI platforms provide the most complete set of passive and active integrated photonic functions including waveguides, splitters, filters, high efficiency grating based fiberchip couplers enabling wafer level testing, phase-shifters, (highspeed) modulators and (high-speed) photo-detectors. For submicron SOI platforms, the waveguide layer is typically only few hundred nanometer thick to ensure single-mode operation of the slab waveguide. The waveguide width is typically between $400 \mathrm{~nm}$ and $1000 \mathrm{~nm}$, and the tight confinement of the optical field in a sub-micron SOI platform allows for waveguide bends of a few microns. For example, single mode strip waveguides in $220 \mathrm{~nm}$ SOI allow for a minimum bend radius of $\sim 5 \mu \mathrm{m}$. Such a small bend radius enables dense integration of waveguide functions on sub-micron SOI platforms.

Over the years, a variety of platforms have emerged with different thickness of the guiding silicon layer [24]-[28], [30], [31], [39]-[44], all with thicknesses of a few hundred nanometer. Even with this variation, SOI with a $220 \mathrm{~nm}$ core thickness has become a kind of de-facto standard and it is being used by a majority of the fabs. Originally this value of silicon thickness was chosen because of its commercial availability in SOI wafers, and it provided a waveguide layer which supported exactly 1 guided slab mode for both TE and TM polarization at wavelengths between $1.5 \mu \mathrm{m}$ and $1.6 \mu \mathrm{m}$. This helped in maintaining single-mode operation in PICs. Moreover, SOI stacks with $310 \mathrm{~nm}, 340 \mathrm{~nm}$ and $500 \mathrm{~nm}$ waveguide thickness have been adopted to facilitate laser integration on SOI [45] [48] and explore mid-IR applications [49]-[52].

The high optical confinement of sub-micron SOI also brings along certain difficulties: the silicon waveguide devices are extremely sensitive to nanometer-scale dimensional variations
[53], [54]. The consequences of this sensitivity are higher waveguide loss, backscattering [55], [56] and spectral shift in interferometric [57] and resonant devices [58], [59]. The propagation losses in sub-micron silicon waveguides can be minimized with thicker and wider waveguides. For example by using $500 \mathrm{~nm}$ thick SOI, waveguides losses of a few $\mathrm{dB} / \mathrm{m}$ are possible to enable applications that require long delay lines, high-Q resonators, narrow filters, etc. [60]. Given that every fabrication process introduces some variability at the nm-scale (inter-wafer, intra-wafer, inter-die or intra-die, both systematic and random), this variability is a fundamental challenge of the sub-micron SOI platforms [61]. However, smart design strategies have been a topic of active research to make fabrication tolerant sub-micron SOI PICs [62], [63]. Another consequence of the high optical confinement in sub-micron SOI waveguides is the onset of two photon absorption (TPA) at power levels of few tens of $\mathrm{mW}$ and higher. TPA not only introduces non-linear propagation losses in sub-micron SOI waveguides but also leads to free carrier absorption (FCA) induced loss and index change. Beyond wavelengths larger than $2.2 \mu \mathrm{m}$, the effect of TPA is drastically reduced [64]. This makes sub-micron SOI structures ideal for implementing non-linear optical devices at these longer wavelengths [49], [65], [66].

2) Thick SOI: For the so-called thick SOI platforms, the waveguide layer is typically thicker than $1 \mu \mathrm{m}$. Silicon photonics started with the demonstration of low loss waveguides on thick SOI platforms [67]-[69]. Importantly, this platform offered one of the first and the longest lasting silicon photonics product in the form of a VOA for PONs [4].

The single mode condition for thick SOIs waveguides is only met for rib-waveguides with a specific aspect ratio of waveguide width and height [69]. Slab and strip waveguides in thick SOI are invariably multimode (at least at telecommunication wavelengths), but it is possible to keep light in their fundamental mode using adiabatic rib-strip conversion structures [70]. The larger mode field leads to increased optical confinement inside the Si core, making thick SOI waveguides less sensitive to dimensional variation, surface roughness and polarization [71], [72]. It also allows them to handle much higher optical power (even $>1 \mathrm{~W}$ ) before nonlinear effects kick in, and to extend the operating wavelength to the mid-IR [75]. Thick SOI waveguides generally have low propagation losses $(\sim 0.1 \mathrm{~dB} / \mathrm{cm})$ and weak backscattering, making them especially suitable for high performance passive functionalities [29], [74]. The most common SOI thickness is $3 \mu \mathrm{m}$, which is also available in open-access [73].

Due to their weak lateral index contrast, the bend radius for rib waveguides in thick SOI is in the mm-scale, which doesn't support dense integration. Therefore, total internal reflection (TIR) mirrors [76] and Euler bends in multimode strip waveguides [77] have mostly replaced rib waveguide bends in thick SOI. With them, the typical bend radius in thick SOI is now ranging from 1 to $50 \mu \mathrm{m}$ [77], which makes also thick SOI suitable for very dense integration. The Euler bends have very low loss for both polarizations $(<0.1 \mathrm{~dB} /$ bend even for a few $\mu \mathrm{m}$ radius) and have enabled implementation of polarization independent devices with compact footprint for optical commu- 
nication [78] and microwave photonics applications [79]. Unlike sub-micron SOI, where high-speed modulation and detection functions are monolithically integrated, open-access thick SOI platforms typically rely on flip-chip-like integration to provide these functions [80]. Nevertheless, low speed modulation of optical signals in the $\mathrm{kHz}$ and $\mathrm{MHz}$ range is available in openaccess thick SOI platforms [29], [81]. High-speed modulators and photodetectors have been reported using thick SOI in [82]-[84] but they are currently not offered by open-access platforms. Input and output coupling for thick SOI waveguides is typically implemented via end-fire coupling or up-reflecting mirrors.

The main limitations in thick SOI technology relate to the large topography of the wafers. This makes it more difficult to realize high-speed active components, to precisely control the waveguide dimensions and to use high-resolution lithography for narrow waveguide features. Due to the high topography, thick SOI PICs are typically fabricated in older generations of CMOS electronics fabs or in MEMS fabs, rather than state-ofthe-art CMOS fabs.

\section{B. Monolithic silicon photonics-electronics co-integration}

Due to the potential compatibility of silicon photonics with the well-established industrialization methodologies used in CMOS technologies, there has been a significant thrust for monolithic integration of silicon photonics with electronics [85]. The additional parasitic capacitance and inductance of wirebonds or micro-bumps used by the hybrid electronic-photonic integrated circuit (EPIC) platforms [86]-[89] is minimized by Front-end-of-line (FEOL) integration of photonic components with electronics [30], [90]. Monolithic EPICs have a potential to meet the stringent power dissipation and aggregate throughput demands for short-to-long-range photonic interconnects [30], [44], [90].

Various demonstrations of monolithic EPICs have been reported [5], [27], [30], [41], [44], [91]-[96]. These implementations are carried out on different types of material platforms such as on bulk CMOS wafers [41], [91], sub-100 nm SOI [30], [44], [93] and $220 \mathrm{~nm}$ SOI platforms [27]. Moreover, various technology nodes such as $90 \mathrm{~nm}$ CMOS [94], $65 \mathrm{~nm}$ CMOS [92], $45 \mathrm{~nm}$ CMOS [93], $28 \mathrm{~nm}$ CMOS [97] and $250 \mathrm{~nm}$ BiCMOS [96], [98] are used for the implementation of monolithic EPICs.

These solutions can be categorized into two approaches. In one approach the transistor process development is completely decoupled from the photonic process development. This approach provides the opportunity to optimize the performance of photonic building blocks as reported in [5], [44], [91], [94] where bulk CMOS or sub-100 nm SOI platforms are used. As an example, the sub- $100 \mathrm{~nm}$ SOI platform demonstrated in [44], [94] provides passive and active functions in the C-band. Similarly, on bulk CMOS platform, in [41], [91], [97] photonic functions are defined on the poly-crystalline silicon layer grown on the locally thicker silicon oxide isolation trenches of the transistor. This platform provides tight bending radius for waveguides and the optical propagation loss in the O-band is $\sim 20 \mathrm{~dB}$ when both active and passive devices where defined on the same wafer. An implementation on $220 \mathrm{~nm}$ SOI platform where optical device modules were inserted in the baseline BiCMOS flow are reported in [27]. In this approach bulk silicon regions on the SOI substrate are defined for the monolithic integration of high-speed electronics and photonics using $250 \mathrm{~nm}$ BiCMOS technology.

The second approach does not deploy any change in the existing CMOS electronics process flow to demonstrate photonics functions. This approach is also known as "Zerochange" silicon photonics [30], [93] and typically uses a sub-100 nm SOI platform. As an example, in [30] such a "Zero-change" silicon photonics platform is demonstrated on a partially depleted SOI providing active and passive photonics functionality with tight bending radius and and low waveguide loss for the C-band after locally removing the silicon substrate under the buried oxide layer, which is not thick enough to prevent optical leakage into the substrate.

\section{Silicon Nitride}

The lower wavelength limit of SOI transparency is $<1.1 \mu \mathrm{m}$, corresponding to the bandgap of silicon. This implies that SOI is not a viable material system for applications operating at visible and very-near-infrared wavelengths. However, inspired by the success of SOI, there has been a growing interest in recent years to extend the wavelength range of "silicon photonics" to the visible domain using silicon nitride ( $\mathrm{SiN}$ ) as a waveguide material. SiN-based silicon photonics [99]-[102] has a transparency window that goes from $\sim 0.4 \mu \mathrm{m}$ all the way up to mid-IR wavelengths of $4 \mu \mathrm{m}$. SiN technologies can be categorized on the basis of the deposition method used (PECVD and LPCVD) [100], [103], [104], [106]-[110]. The PECVD platforms exhibit higher waveguide losses at the $1.55 \mu \mathrm{m}$ telecommunication band but do not require processing steps at temperature beyond $450^{\circ} \mathrm{C}$, which is an asset for certain process flows and applications. For applications operating at $1.55 \mu \mathrm{m}$ LPCVD-SiN is typically used. Because there is no TPA in SiN, much higher power densities than in submicron SOI can be supported, and the lower loss waveguides (with lower phase errors) make it easier to create high-quality filter circuits [111], [112].

SiN provides moderately high index contrast, which is 3.5 to 4.5 times less than that of sub-micron SOI in the telecommunication window. This leads to reduced loss and an order of magnitude weaker back-scattering for the $\mathrm{SiN}$ based waveguides [101], [113]. As compared to sub-micron SOI platforms, $\mathrm{SiN}$ has $\sim 5$ times lower temperature sensitivity [101]. This means that $\mathrm{SiN}$ is a better suited platform for applications in which the temperature sensitivity may be an issue, but where active temperature control is not desirable. At the same time, the low temperature sensitivity of SiN leads to a higher power dissipation for tunable devices using the thermo-optic effect. Similar to SOI, we can identify trends towards thin and thick SiN core layers. Typically thick SiN layers are exploited to develop high-power silicon photonics PICs [114]. This diversity of layer thickness is enabled by the flexible PECVD or LPCVD process used for the deposition of $\mathrm{SiN}$ layers. This contrasts with the SOI case, where the SOI 
wafer provider will only manufacture wafers of a specific layer thickness if there is a sufficient demand for it.

\section{Germanium based silicon photonics}

$220 \mathrm{~nm}$ SOI with $2 \mu \mathrm{m}$ BOX has a transparency of up to $2.9 \mu \mathrm{m}$ [115] whereas this transparency reaches $3.8 \mu \mathrm{m}$ for SOI with $400 \mathrm{~nm}$ guiding silicon layer thickness [50], [116], [117]. For the former, the transparency is limited by the substrate leakage while for the latter, the transparency is limited due to the onset of absorption in the buried oxide layer of SOI. Approaches such as the implementation of suspended SOI strip and slot waveguides are developed to extend the transparency range further into the mid-IR wavelengths [118]-[121].

Germanium-on-silicon (GOS) provides a moderate-verticalcontrast waveguide system with a transparency window in the mid-IR range of $2 \mu \mathrm{m}$ to $8 \mu \mathrm{m}$ [117], [122]-[125]. Though Germanium itself is transparent up to $\sim 14 \mu \mathrm{m}$, the silicon cladding starts absorbing at $\sim 8 \mu \mathrm{m}$ and beyond. This makes it a suitable silicon photonics material systems for on-chip spectroscopic systems for gas and liquid sensing, monitoring of air or oil quality, control of engine emissions, free-space communication and light detection and ranging (LiDAR) systems [117], [122], [126]. Apart from GOS, there has been a variety of other Germanium-based mid-infrared technology platforms such as Germanium-on-SOI [127]-[129], Germanium-on-Silicon Nitride [130], $\mathrm{Si}_{(1-x)} \mathrm{Ge}_{x}$-on-Silicon [131] and suspended Germanium [132], [133].

Figure 4 gives an overview of the various single-mode waveguide cross-sections possible with the different flavours of silicon photonics platforms discussed above. The sub-micron SOI and SiN platforms support fully etched strip waveguides, deep and shallow etched rib waveguides, and slot waveguides. Specialized geometries for waveguides such as Box-shell are also available in SiN-based silicon photonics [104]. Both sub-micron SOI and SiN material systems support different types of claddings (i-e, air and oxide). Thick SOI supports only rib waveguides with different cladding types, whereas germanium-based mid-infrared platforms typically support aircladded waveguides of strip, rib and slot types. Fig. 5 shows the transparency range of SOI, SiN and GOS material systems and the corresponding applications linked to optical transparency windows of these material systems.

\section{E. Need for standardization in silicon photonics}

Each fab requires to develop a standardized technology with an established and mature process flow to provide manufacturing of silicon photonics PICs. If more fabs adopt the same standard, fabless companies will have more supplier options, which reduces the costs and the risks for the silicon PIC design. However, in the current landscape of silicon photonics technologies, there is no single standard technology. Rather, each fab has defined its own standard technology. This helps them to maintain fewer processes, which results in reduced cost for a foundry.

Due to the high index contrast of the material system, dimensional control is important [31], [54], [57]. For example, the process control in terms of waveguide width and

\begin{tabular}{|c|c|c|c|c|c|}
\hline $\begin{array}{l}\text { Waveguide } \\
\text { Type }\end{array}$ & $\begin{array}{c}\text { Single Mode } \\
\text { Waveguide Cross- } \\
\text { sections }\end{array}$ & $\begin{array}{c}\text { Sub- } \\
\text { micron SOI }\end{array}$ & Thick SOI & $\mathrm{SiN}$ & $\begin{array}{l}\text { Ge-based mid- } \\
\text { infrared material } \\
\text { systems }\end{array}$ \\
\hline \multirow{2}{*}{ Strip } & Air & $\checkmark$ & 冈 & $\checkmark$ & $\checkmark$ \\
\hline & & $\checkmark$ & 冈 & $\checkmark$ & 冈 \\
\hline \multirow{2}{*}{ Rib } & Air & $\checkmark$ & $\checkmark$ & $\checkmark$ & $\checkmark$ \\
\hline & 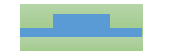 & $\checkmark$ & $\checkmark$ & $\checkmark$ & 冈 \\
\hline Slot & Air & $\checkmark$ & 冈 & $\checkmark$ & $\checkmark$ \\
\hline Box-shell & $\square$ & $\boldsymbol{x}$ & 冈 & $\checkmark$ & 冈 \\
\hline
\end{tabular}

Fig. 4: Typical cross-sections of single-mode waveguides supported by the different variants of silicon photonics platforms. In many cases the air cladding is replaced by a silica cladding, which creates a less fragile waveguide (less susceptible to dust).

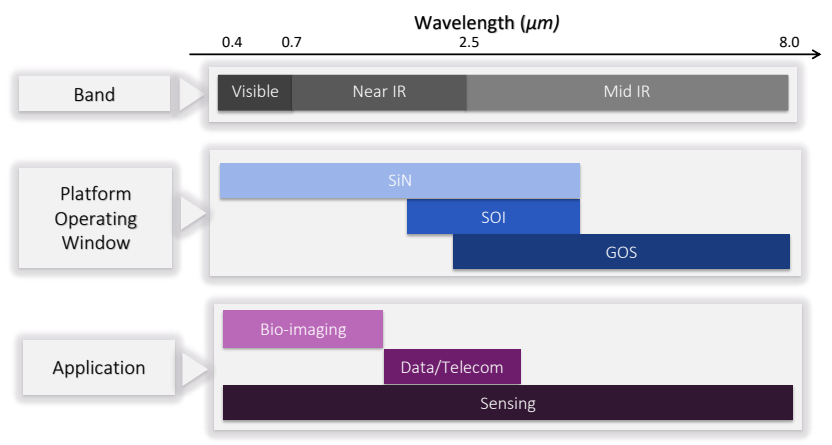

Fig. 5: Different forms of silicon photonics operating from visible to mid-IR wavelengths to provide integrated photonics solutions for bio-imaging, optical communication and sensing.

height required for wavelength selective devices in sub-micron SOI devices is more stringent than for CMOS electronics. Standardization in the process flow provides stability and repeatability in the device performance for large manufacturing volumes. Moreover, it helps in gauging variance in key device performance parameters [24], [31]. A well-understood and stable device performance is crucial to develop complex photonic integrated systems on a chip.

\section{F. Value of diversity in silicon photonics}

A single standardized technology platform is not necessarily capable of addressing the requirements for various applications in the very wide range of market sectors where PIC technology can potentially bring new value [134]. This means that, while standardization is a must for the above-mentioned technical and economic reasons, so is the diversification. This is not something that is unique to silicon photonics. Again, we can draw the comparison with electronics, where CMOS is the most dominant technology, but which comes in a variety of nodes with different performance and price points. On top of that there is diversity for targeting specialized markets such as high-power electronics, ultra-high frequency RF electronics, ionization radiation resistant (rad-hard) electronics, etc. 


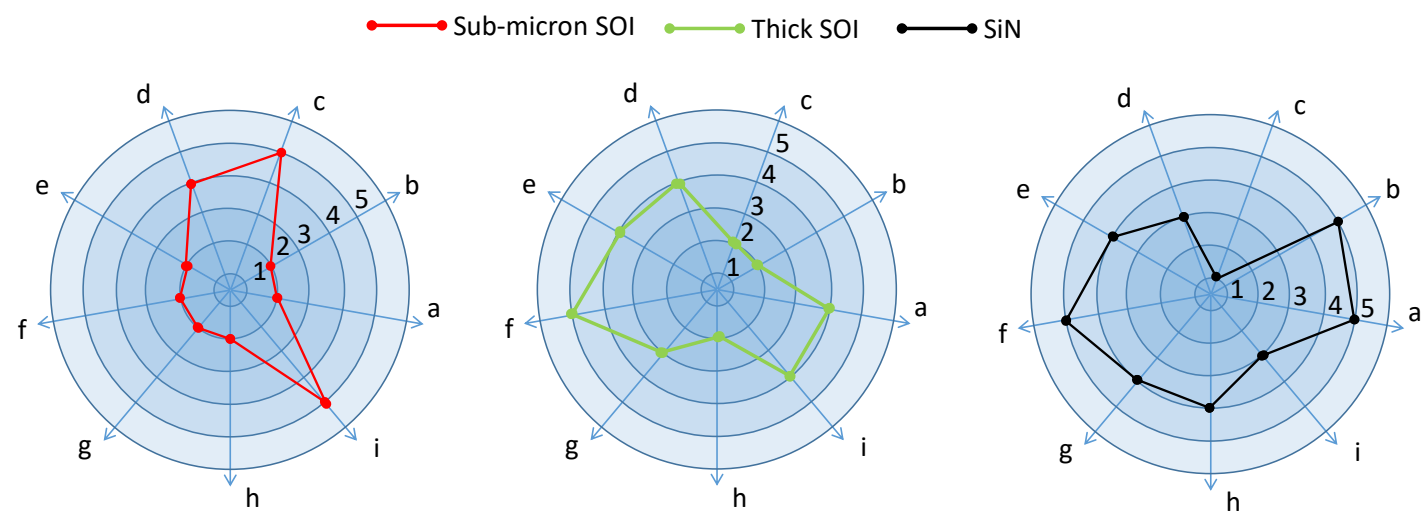

(a) Propagation Loss, (b) Layer Stack Flexibility, (c) Active Functions, (d) Efficient IO, (e) Back-scattering, (f) High power operation, (g) Transparency, (h) Thermal sensitivity, (i) Bend Radius

Fig. 6: Qualitative representation of the current status of sub-micron SOI (in red), thick SOI (in green) and SiN (in black) on a scale of 1 to 5 (5 is better). Sub-micron SOI complements SiN in providing compact form factor PICs through its small bending radius, high-efficiency IO by using grating/edge couplers and active functionalities such as high-speed photodiodes and detectors. On the other hand, SiN provides low loss waveguides, broad transparency window for visible, NIR and midIR applications, ability to deal with 100s of $\mathrm{mW} \mathrm{CW}$ power and reduced back-scattering due to its lower index contrast than SOI. Thick SOI is known to provide lower propagation loss, lower back-scattering, and high power operation but does not provide high-speed active functionalities.

The degree of material diversity in silicon photonics enables a situation where technology platforms complement one another. Such a diversity not only prevents monopoly of one technology platform but also develops a sense of competition between technologies and makes them agile for evolution. As an example, Fig. 6 provides a qualitative comparison of sub-micron SOI (left), thick SOI (middle) and SiN-based (right) silicon photonics platforms. One can observe the complementarity of $\mathrm{SiN}$ (right plot of Fig. 6) and sub-micron SOI (red lines in left plot of Fig. 6). SOI complements $\mathrm{SiN}$ by providing compact form factor PICs through its small bending radius, highefficiency fiber-chip coupling by using grating/edge couplers and active functionalities such as high-speed photodiodes and detectors. On the other hand, SiN provides lower loss waveguides, broad transparency window for visible, NIR and mid-IR applications, ability to deal with 100s of mW CW power and reduced back-scattering due to its lower index contrast than SOI.

Table I lists the type of material stacks used by various openaccess silicon photonics technologies and their key technical differentiators. Moreover, it is also possible to combine elements of different platforms together. The manufacturing flexibility of SiN layers makes it possible to integrate SiN layers into the SOI platforms [108], [135]-[139]. In such a platform, passive components demanding low loss and high fabrication tolerance are defined on the SiN layer [140], [141]. Functions such as modulators and photodiodes are then defined in the Si layer.

Along with the platform diversity there is a diversity in infrastructure needed for silicon photonics. Most of the submicron SOI platforms use $200 \mathrm{~mm}$ or $300 \mathrm{~mm}$ wafers. For NIR applications on sub-micron SOI, the desired feature sizes or critical dimensions (CD) can be delivered by $130 \mathrm{~nm}$ or
$90 \mathrm{~nm}$ CMOS pilot lines and foundries. However, due to the high index contrast of sub-micron SOI, the required accuracy corresponds more to a $40 \mathrm{~nm}$ or $65 \mathrm{~nm}$ CMOS technology node. These nodes already make use of the high-end $300 \mathrm{~mm}$ manufacturing equipment such as immersion lithography, do not yet require expensive techniques such as double/triple patterning. Still, the processing cost on $300 \mathrm{~mm}$ wafers at these nodes is considerably higher than that of older CMOS nodes on $200 \mathrm{~mm}$ wafers. Yet, for mid-infrared PICs the scaling - with wavelength - of photonic structures to larger dimensions relaxes the fabrication tolerances for sub-micron SOI and GOS based PICs. Due to the relaxed tolerances of moderate index contrast material systems (i-e, SiN, GOS), the platforms on these material systems demand less advanced lithography and etching infrastructure, making them more cost-effective in certain volume manufacturing cases. As a result, wafer sizes of $100 \mathrm{~mm}, 150 \mathrm{~mm}$, and $200 \mathrm{~mm}$ are still prevalent today for these platforms.

In the current landscape of open-access technologies, there is clear scope for a moderate number of diversified platforms. The sustainability of these platforms depends on whether there is sufficient market for each platform. Therefore there is a "sweet spot" in terms of standardization and diversity. It is still an open question how this sweet spot will evolve and which silicon photonics platforms will prove to be sustainable.

\section{The Silicon Photonics Open-Access Model}

\section{A. Access Models}

One of the key mechanisms behind the economic success of silicon electronics is the open-access model, where fabless companies can have chips fabricated in commercial foundries that provide standardized fabrication platforms. This separation of design and product development on one hand, and fabrication 
on the other hand, has acted as a multiplier in economic activity, with hundreds of thriving fabless companies for every foundry [34], [142], [143]. It is a win-win formula, lowering the investment for fabless companies and generating profitable volumes for the foundries. In the silicon electronics ecosystem, the open-access foundry model can perfectly coexist with vertically integrated IDMs that benefit from a tight integration of product know-how and their own proprietary fabrication processes.

Given the many parallels between silicon photonics and silicon electronics, the open-access foundry model (or fabless model) can stimulate a similar growth in fabless PIC-based product development. Open-access is a model that offers fabrication services to third parties i.e. to external users /clients outside a technology consortium. open-access is facilitated through an eco-system providing appropriate support services and tools such as design software, packaging, and testing [34], [144]-[146].

Open-access is not the only possible access model to fabrication technology. We already mentioned IDMs with vertically integrated fabrication and product development, but it is also possible for fabless companies to access foundry capabilities on a bilateral or multilateral basis, co-developing proprietary flavors of a foundry's technology platform. This is a model that has yielded some of the most visible successes in silicon photonics [37], [38], [90], [147]. These are models that we also find in electronics and other custom semiconductor platforms such as micro-electromechanical systems (MEMS). Fig. 7 gives a schematic representation of different access models for PIC-based product development.

Given that a fabless/foundry ecosystem should address the largest possible application space, the versatility of silicon photonics open-access technology platforms and the ease of their access is of paramount importance. This allows fabless companies to develop innovative products and evolve seamlessly from prototyping to small-volume manufacturing and possibly all the way to high volume manufacturing [142]. Like in electronics, this will also require a certain diversity in the technology offerings, allowing product developers to pick the platform best suited for their application.

\section{B. Open-Access Workflow}

In an open-access model, it is important for all parties involved that fabless companies find their way to a suitable foundry as easily and as affordably as possible. For electronics, this was given a boost through the creation of the multi-projectwafer service MOSIS in 1981 [23], [34], [144]-[146],later mirrored by Europractice IC in Europe [148]. For silicon photonics, ePIXfab - a joint initiative by imec (Belgium) and CEA-LETI (France), operated from Ghent University (Belgium) - pioneered a similar access mechanism for silicon photonics through MPW service since 2006. By 2011, the open-access silicon photonics technologies of IHP and VTT were also

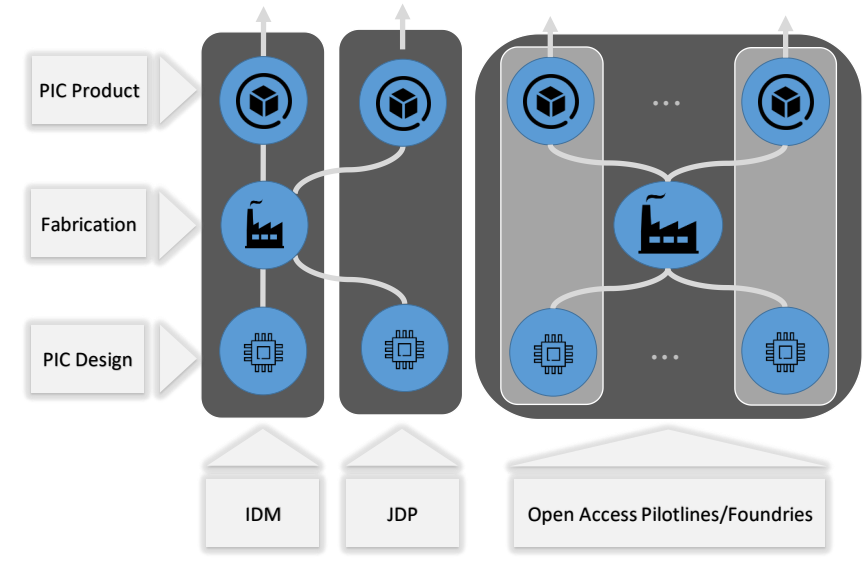

Fig. 7: Schematic representation of three prominent access models for PIC manufacturing. The first column represents vertically integrated IDMs. They internally control the entire process from chip design to production. Fabless companies can have joint development programs (JDPs) with IDMs as represented in the second column. The third column shows different end-users submitting their PIC designs to openaccess silicon photonics pilot lines/foundries for their product development.

\section{offered by ePIXfab. ${ }^{1}$}

A similar initiative, named Optoelectronic Systems Integration in Silicon (OpSIS), was launched in 2010 from University of Washington and University of Delaware [38]. While the initiative did not sustain beyond 2015 it was vividly successful in providing design support for researchers in silicon photonics and organization of multiple MPW runs through fabs such as BAE Systems CMOS fab and Institute of Microelectronics (IME) in Singapore [37], [38]. Recently, a new initiative called the American Institute for Manufacturing integrated Photonics (AIM Photonics) has emerged. It is currently engaged in establishing an ecosystem supporting the complete silicon photonics product development (from design to prototype to pilot or mid-scale wafer and package manufacturing) using the $300 \mathrm{~mm}$ fab at the State University of New York (SUNY) Polytechnic Institute and packaging facilities in Rochester, New York [149], [150].

Various other initiatives, which provide open-access silicon photonics technologies to end-users have also emerged. For example, Photonics Electronics Technology Research Association (PETRA) in Japan and Electronics \& Telecommunications Research Institute (ETRI) in Korea provide access to the end-users in these respective countries. Similarly, other countries have developed open-access technologies to address the demands of local and/or global end-users.

When an end-user wants to access PIC fabrication technology, the first challenge is to determine if silicon photonics technol-

\footnotetext{
${ }^{1}$ Since 2015, the MPW services offered by $e P I X f a b$ have been transferred to Europractice IC Service. ePIXfab has transformed itself into the European Silicon Photonics Alliance, with the majority of the European open-access silicon photonics fabs as its members, and continues to promote silicon photonics science, technology, and application through fabless models (http://epixfab.eu).
} 
ogy will meet the system specification and which technology flavors from a diverse pool of foundries are the most suitable. In many cases, an end-user product developer does not have the internal technical know-how to make this platform selection, let alone kick-start the design of a full-custom complex PIC As with custom electronics, this creates a space for photonic design houses and brokers that can support the end-user in his choice of technology.

After the selection of technology, the open-access process makes it possible to get started on a practical design without the need for complex bilateral discussions. Open-access foundries provide access to process design kits (PDK) after signing a standard design kit license agreement (DKLA) with the fab. In a case where the PIC design is outsourced to a design house, a similar non-disclosure agreement (NDA) is usually signed with the design house.

The PDK provided by the foundry is the interface between the designer and the technology [151], [152]. It contains sufficient technical details of the technology to enable the designer to create either full-custom layouts or custom circuits. A PDK is typically compatible with specific design tools and is always foundry-specific. Unlike with electronics, where there is some standardization of the content of PDKs, there is as yet no standardized blueprint for photonics PDKs. In its simplest form, it contains layer description associated with a process step and critical dimensions that define the design rules for the process steps. More advanced versions of PDKs also contain:

- Component libraries with their parametric cells and their layouts (either actual layouts or IP-protected black-boxes)

- Technology handbooks describing different technology steps and process variability statistics, design rule manual and component library handbook providing statistics about their performance

- Design rules enabling the designer to match the critical dimensions and density of the design with the capabilities of the fab concerning mask preparation, lithography and chemical-mechanical polishing. These design rules are often embedded in a deck for design rule checking (DRC) software.

Recently fabs and design tool developers have started codeveloping calibrated compact model libraries (CML) for the PDKs of their respective open-access technologies. The CML is meant to improve the accuracy and reliability of PIC designs by enabling the designers to accurately simulate and optimize the performance of complex PIC designs before fabrication [153], [154]. In the future, such CMLs will be an integral part of PDKs. Furthermore, the fabs have also been striving to include 3-D capable layout-vs-schematic (LVS) checking and the capability to run co-simulation with electronics ICs [61].

\section{Open-Access Modalities}

Generic open-access technologies are available in different modalities, depending on the phase of the product development:

- Multi-project wafer (MPW),

- Dedicated engineering runs,

- Pre-production, low and high volume manufacturing.
For the proof-of-concept and early stage $\mathrm{R} \& \mathrm{D}, M P W$ shuttle runs are ideally suited. MPWs play an instrumental role in catalyzing the field of silicon photonics by providing lowcost access to start-ups, small/medium enterprises (SME), and low-CAP companies to test their design ideas using a standard process flow of different fabs offering open-access technologies [33], [34], [143]. MPW helps the designers to gauge the capabilities available in a technology. In MPWs, several users share the design area and share the mask and fabrication cost. Typical design sizes for each user consume few tens of $\mathrm{mm}^{2}$ with a typical cost of a few $\mathrm{k} €$ for passive PICs to a few tens of $\mathrm{k} €$ for PICs with advanced functionalities of modulation and photo-detection [148]. In this stage, the end-user typically receives few dozens of chips. The designer has the freedom to make the design using custom components from his own component library and a component library of the fab PDK. The intellectual property (IP) of the user is preserved by ensuring that the designs of the user are not visible to any other user sharing the same design reticle.

In many cases, the fab provides access to MPW prototyping services through a technology broker. Due to the very lowvolume nature of the MPW service, fabs collaborate with technology brokers who aggregate the designs of the end-users. Moreover, they support the end-users by providing access to PDK for the fab, relevant design tools, and undertake layout verification by design rule checking (DRC). A technology broker can provide support for multiple technology platforms from different foundries. Table I lists some of the most prominent open-access silicon photonics platforms offered by various CMOS pilot-lines, research institutes and industrial fabs.

On average each foundry offering MPW shuttles provides three to six runs in a year. In most cases, the MPW access is provided by research fabs. These fabs typically have a long fabrication cycle due to the limited capacity of their infrastructure. Typically, the turn-around time for a design with full process flow (e.g., passives and actives) takes 9-12 months. If the design has only passive devices, then this time is typically 4-6 months. These long production cycles prohibit the rapidlearning desirable during the early research, development and prototyping phase of the product development. To circumvent this very issue, rapid prototyping services for silicon photonics have emerged. In some cases, these prototyping services are compatible in terms of processes, specifications, design flow and design rules with the technology offered by an MPW fab. The typical turn-around time for a rapid prototyping service is few weeks whereby an end-user gets one to ten chips by paying a cost comparable to an MPW run through a fab. Most rapid prototyping platforms provide passive functions only, but in a few cases, they offer the complete process flow comprising both active and passive functions. Table I lists the few prominent platforms available in rapid-prototyping mode.

Typically 50-70\% of end-user designs in an MPW are based on user-defined custom building blocks [151]. Before moving to early prototype dedicated production runs, typically an end-user requires multiple tape-out iterations to optimize custom-designed building blocks and reach the performance specifications. This cycle of design optimization is not only 
TABLE I: Prominent open-access silicon photonics technology platforms offered by various fabs, their technology and access mechanism.

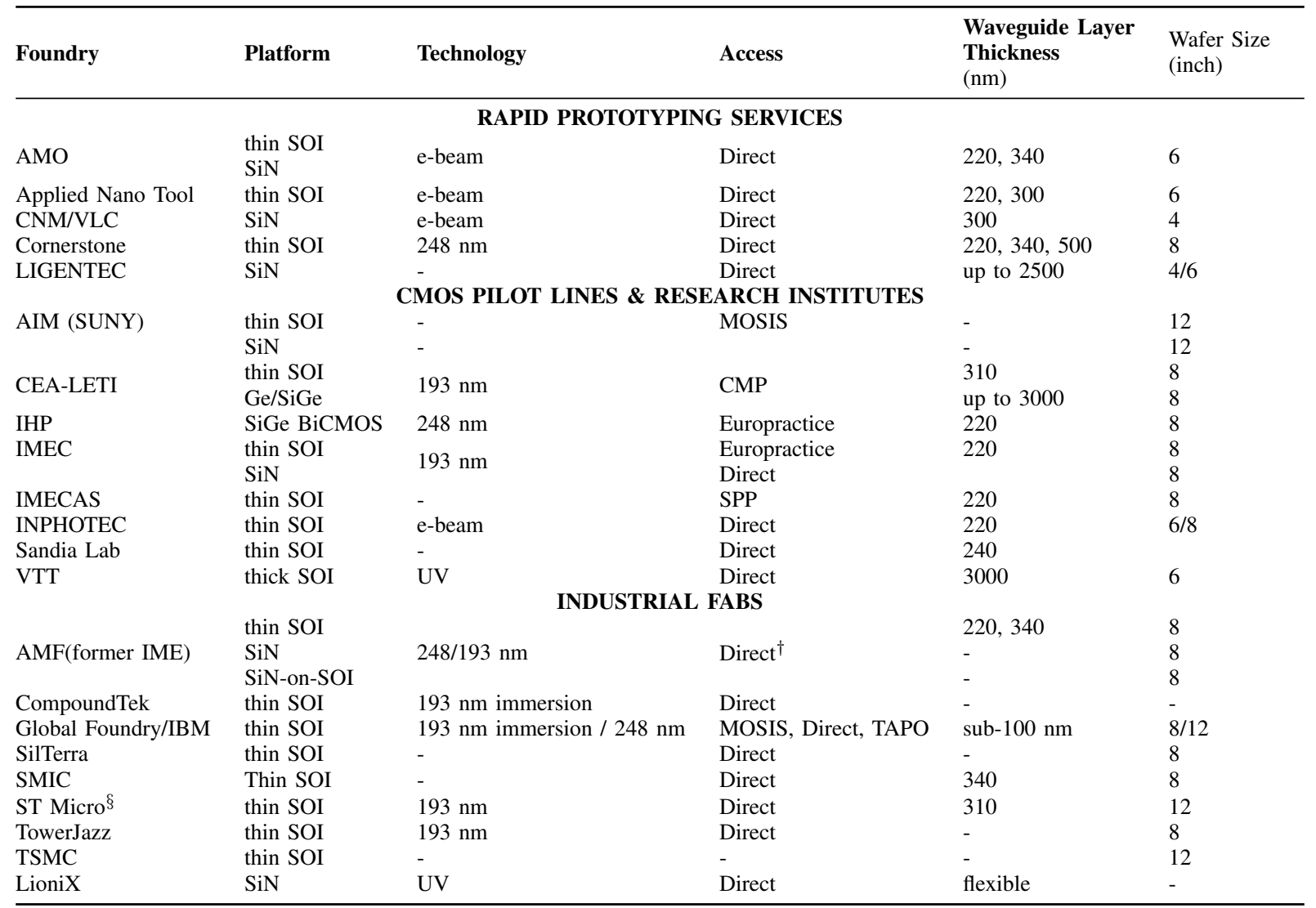

†CMC Microsystems (Canada) brokers MPW services to Canadian universities and industries.

$\S$ ST Micro platforms is accessible via bilateral contracts

time consuming but also has a significant economic cost. An alternative is to go for a dedicated engineering run directly. In such tape-outs, the same process flow as for MPW shuttles is used, but a user can get more wafers and more design space. While expensive, they provide the end-users with an opportunity to put large custom building block design sweeps to optimize the overall PIC design with potentially fewer iterations.

Silicon photonics technology accessible via MPW has a fixed process flow with typically a high level of maturity (TRL level 6 and beyond). The platform is generic in the sense that slight variants of the same process flow may be used for diverse applications. Despite the generic nature of open-access technology, in many cases this process flow is incapable of delivering the desired performance specifications. Also, when moving from R\&D to commercialization, a generic platform might still fall short of the functional performance specifications of a PIC through design optimization only. Both cases demand a customized process flow, which may involve new process development through dedicated engineering runs. Various iterations are carried out to achieve maturity for the customized processes, which makes the turn-around time of such dedicated engineering runs longer than is the case with MPW. The end-users are provided with a customized PDK with agreement on the design-rules and design layers making these runs more flexible. Typically a customized dedicated engineering run costs $10 \times$ more than an MPW participation.

Once the PIC design has reached a certain maturity, the silicon photonics product development requires pre-production engineering runs. During these runs, the process repeatability is established to ensure a predictable process flow. During this phase, corner lots are run as a critical validation step to determine the worst-case estimate of the system functional performance specification. Such lots are important for defining the ideal process conditions for low or high-volume manufacturing. Manufacturing volumes of a few hundreds to a few thousands of wafers per year are considered to be lowvolume for PIC manufacturing. As today most open-access technologies are offered by $R \& D$ fabs, this is the only volume bracket that can be addressed, as these $\mathrm{R} \& \mathrm{D}$ fabs generally do not have a capacity for medium- or high-volume production. However, R\&D fabs may have agreements where the endusers can translate the process developed in a R\&D lab to commercial fabs providing medium-and high-volume manufacturing. This process transfer requires matched geometries (i-e, minimum feature size), a similar performance of building blocks including the performance variability, matched PDK and associated device models, and migration of other parts of the supply chain (e.g., assembly). ST Microelectronics, though an IDM, is one of the first foundries to provide access to its technology platform for volume manufacturing through 
bilateral contracts [147]. Other foundries with the capacity for high-volume manufacturing such as GlobalFoundries and TowerJazz have also announced to launch open-access silicon photonics platforms. Moreover, in 2017, TSMC developed a through-silicon-via (TSV)-enabled silicon photonics platform in a $300 \mathrm{~mm}$ foundry. This platform has demonstrated stateof-the-art performance for various passive and active building blocks [90].

Table I provides a non-exhaustive list of institutes and foundries providing (or in the process of providing) open-access silicon photonics technologies. In the early days, the fabs chose $220 \mathrm{~nm}$ SOI as the substrate for their platform development [24], [25], [27], [31], [41]. More recently silicon photonics open-access platforms based on $310 \mathrm{~nm}$ SOI [26], [40], $500 \mathrm{~nm}$ SOI [155], SiN and Germanium-based mid-infrared platforms have also emerged. The technology, access mechanism and wafer size used are also listed in Table. I. The table will likely evolve considerably in the next years.

\section{Recent Open-Access Silicon Photonics Technology and Eco- system Developments}

1) Process technology: Advances in process technologies for silicon photonics platforms has resulted in their improved performance. These improvements resulted in demonstrating passive devices with lower loss, superior spectral accuracy and higher reproducibility. Performance of active devices has also benefited from the evolved processing technology whereby different types of efficient modulators and photo-detectors operating at high-speed are demonstrated [24]-[28], [31], [32], [156]-[161]. As a result of advances in the process technology, open-access fabs have consolidated their platforms by offering new process modules such as very low loss waveguides [156], [157], high-speed modulators [162]-[167], integration of efficient electro-optic materials in a silicon photonics process flow [174], [175], multi-layer SiN on Si [108], [135]-[139], and broadband fiber-chip-fiber couplers [168]-[173], [176].

Most applications of PICs require that a light source supply the chip with either a clean optical carrier (laser) or broadband light. A variety of approaches are pursued to bring III-V and SOI-based silicon photonics together [177]-[179]. Demonstrations are reported to reach the ultimate solution of a monolithically integrated quantum-well or quantum-dot III-V laser source in Silicon Photonics [180]-[186]. Currently, the most mature solutions are provided by hybrid or heterogeneously integration of quantum well or quantum dot laser source with silicon photonics PICs [184], [187]-[189]. Openaccess platforms are actively engaged in developing the waferscale integration of light-sources on silicon PICs. Wafer-scale integration is required to preserve the cost advantage of silicon photonics. A recent demonstration of wafer-scale heterogeneous integration of light-sources is reported in [190]. An incumbent technology that can provide wafer-scale integrated laser sources is micro-transfer-printing ( $\mu \mathrm{TP})$ [191]. Other open-access technology consortia such as AIM Photonics are also exploring the development of integrated laser solutions including quantum dot based wafer-scale epitaxially grown laser sources within their silicon photonics technology platform [149], [150].
To gauge the process stability and capability of the PIC platforms offered by the open-access fabs, methodologies and infrastructure for platform performance tracking are developed by monitoring the key dimensions for each processing step [24], [192]-[194]. Furthermore, infrastructure for wafer-scale end-of-line optical, electro-optic and electrical testing using automatic setups is set up to determine device performance [31], [90], [195].

2) Design Tools: Open-access technologys only make sense in combination with a design infrastructure which enables platform users to design their own devices and structures. We can identify two essential parts of the design infrastructure: design/simulation tools and process design kits (PDK). A number of design tools for photonic integrated circuits have emerged in the past two decades [152]. At first, they supported the designer in generating complex layout features for photonic components, which are often much more complicated than those for electronics. Photonics requires curvilinear waveguides and custom all-angle geometries, while typical electronic layouts are based on simple rectangular features. The resulting geometries can be simulated using electromagnetic solvers, which can be quite computationally heavy.

Today, photonic design is gradually migrating to the circuit level [152]: instead of defining and simulating every individual geometry, a photonic circuit is constructed of building blocks and connected with waveguides. Each building block has an efficient compact model which is used to calculate the reponse of the entire circuit. This is similar to electronic circuit design, where compact models are defined in SPICE or VerilogA. Photonic models can also be defined in VerilogA [196], or in a model for a specialized photonic circuit simulator. Such circuit-level design, which starts with a schematic and circuit simulation, and only then implements the circuit as an actual layout, can scale up to designs of much larger complexity. Also, circuit-level design allows the user to focus on the added value of his application, rather than redesigning low-level functional building blocks.

Circuit design requires a library of components that can be connected together into a circuit. For each technology platform, fabs supply a PDK which contains a standard component library for essential functions such as waveguiding, splitting, crossing, modulation, detection and fiber coupling. Until recently, these component libraries consisted of simple layouts that a designer could reuse at the circuit level, but the PDK libraries are now gradually populated with compact models for the building blocks, enabling designers to verify the circuit function in simulation.

Most photonic design tools are available as commercial software. Unfortunately, at this point there is only limited interoperability between tools of different vendors, even though some collaboration and standardization activities are emerging [197]. This also extends to integration of photonic design tools and electronics design automation (EDA) tools, as we see EDA vendors gradually supporting photonic design [198], [199].

Because high-contrast silicon photonics components are so geometry sensitive, it is important that the effects of the fabrication process are taken into account at the design stage. Design for manufacturability (DfM) techniques that originated 
in electronics are now being adapted and extended for photonics. This includes litho-friendly design (LFD) [200], tolerant circuit design [63], use of optical proximity corrections [201] and tiling to control pattern densities [202].

To obtain a first-time-right design, an effective verification strategy is needed. This consists of two steps: Design rule checking (DRC) ensures that the layed-out design can actually be fabricated, while layout-vs-schematic (LVS) tests extract the functional circuit from a layout and compare it to the original design intent, including simulation of the extracted circuit. Robust layout verification flows have also been under development to ensure that both the mask fabrication and silicon processing will not be affected by the inclusion of designs which are not qualified [61].

The landscape of design tools and PDKs is rapidly evolving, with new capabilities for designers being released every few months. This is needed, because a low-threshold design experience is a direct enabler to open-access technology.

3) Packaging: The packaging of silicon photonics chips is of critical importance both at the prototyping and the manufacturing level. Efforts have been made in standardizing the packaging processes to reduce the cost of packaging and to improve the reliability issues associated with optical and electrical interfaces to silicon photonics chips. Passive and automated packaging schemes are developed to cut the cost associated with photonics packaging [203]. Similar to PIC layout design rules, there have been efforts to develop packaging design kits and packaging design rules for costeffective packaging of silicon photonics chips, their thermal management, integration with electronics and assembly of off-chip light sources. Recently, the open-access Photonic Integrated Circuit Assembly and Packaging Pilot line (PIXAPP) has been established in Europe to provide end-users easy access to the packaging of PICs. Similar initiatives are emerging in other continents. For example, the open-access platform of AIM Photonics [149] and the one from Sandia National Laboratories are combined with chip scale assembly and packaging service.

4) Emerging Silicon PIC Prototyping \& Manufacturing Initiatives: The success and impact ePIXfab created by pioneering open-access silicon photonics technologies acted as a blueprint for various other players. With a growing number of initiatives, such as $O p S I S$ and $A * S T A R I M E$, imitated this success and resulted in creating a much broader and active community engaged in PIC-based R\&Development. Though OpSIS has ceased and ePIXfab handed over its brokering role to Europractice and CMP, the ePIXfab members (IMEC, CEALETI, IHP,$V T T$ ) and $A * S T A R$ IME continue to consolidate their respective technologies by the inclusion of new process modules and enhancing their TRLs.

Recent years have seen numerous new initiatives by CMOS pilot lines and public/private research institutes to provide open-access silicon photonics technologies. For example,in Europe, the pilot lines PIX4Life providing PIC prototyping/manufacturing for life-science applications and MIRPHAB for mid-IR applications have surfaced. $A * S T A R I M E$, which is pioneering Asian institute providing open-access silicon photonics, has started a spin-off Advanced Micro Foundry (AMF) in 2017 to provide manufacturing of silicon PICs using the processes and technology developed by $A * S T A R$ $I M E$. In Japan the PETRA initiative, in Korea Electronics and Telecommunications Research Institute (ETRI) and in China IMECAS have started providing silicon PIC platforms to their domestic end-users. In North America, Sandia Research Laboratories and AIM Photonics have made progress to develop and offer silicon photonics platforms. Anticipation a demand for high-volume PIC-manufacturing, CMOS pilot lines and research institutes have developed routes for high-volume manufacturing through commercial fabs. Moreover, commercial fabs have developed silicon photonics PIC platforms and started offering them to third-party end-users.

\section{SUMMARY}

Open-access of silicon photonics technologies, initially offered by CMOS research institutes, has played an instrumental role in making it a mainstream photonic integration technology. Thanks to the continuous consolidation of the technology platforms offered by CMOS pilot lines and research institutes, low-volume manufacturing of silicon photonics PICs is already happening. The number of fabless companies envisaging silicon photonics products and requiring the manufacturing of their products is rapidly increasing. With this clear trend in market growth and looming demand for high-volume manufacturing by fabless companies, pure-play foundries have started offering open-access silicon photonics technologies for prototyping as well as manufacturing at low- and high-volume level.

The potential penetration of silicon photonics into market sectors beyond optical communication further augments the growth of silicon photonics. The flavour of silicon photonics geared towards visible wavelengths and mid-infrared wavelengths respectively have emerged to address life-science and sensing applications. There are cases where the different flavors of silicon photonics compete with each other, making the technology more agile for evolution and there are cases where these flavors complement each other to strengthen silicon photonics against competing technologies. Since silicon photonics is also viable at moderate volumes, many of the existing silicon photonics flavors may grow industrially even if they target specialized applications.

The developments made by the open-access technology platforms have galvanized the silicon photonics eco-system. A mature and complete supply chain from design to packaging and testing is imminent.

\section{ACKNOWLEDGMENT}

The authors would like to thank Dr. Timo Aalto (VTT), Prof. John Bowers (University of California, Santa Barbara), Dr. Chris Doerr (Acacia Communications, Inc.), Dr. Jean-Marc Fedeli (CEA-LETI), Dr. Patrick Lo Guo-Qiang (Advanced Micro Foundry), Dr. Amit Khanna (Elenion Technologies), Prof. Thomas Koch (The University of Arizona), Prof. Joyce Poon (University of Toronto), Prof. Hon Ki Tsang (The Chinese University of Hong Kong) and Prof. Lars Zimmermann (IHP) for their kind inputs to improve the quality of this manuscript. 


\section{REFERENCES}

[1] M. Salib et al., Silicon Photonics, Intel Technology Journal, Vol. 8, Issue 2, p143-160, May 2004.

[2] W. Bogaerts et al., Nanophotonic waveguides in silicon-on-insulator fabricated with CMOS technology, Journal of Lightwave Technology, vol. 23, no. 1, pp. 401-412, Jan. 2005.

[3] B. Jalali and S. Fathpour, Silicon Photonics, Journal of Lightwave Technology, vol. 24, no. 12, pp. 4600-4615, Dec. 2006.

[4] A. Rickman, The commercialization of silicon photonics, Nature Photonics, Volume 8, 2014.

[5] A. Huang et al., A 10Gb/s photonic modulator and WDM MUX/DEMUX integrated with electronics in 0.13/spl $\mu \mathrm{m}$ SOI CMOS, 2006 IEEE International Solid State Circuits Conference - Digest of Technical Papers, San Francisco, CA, 2006, pp. 922-929.

[6] K. Greene, Silicon Photonics Comes to Market, MIT Technology Review, https://www.technologyreview.com/s/408520/ silicon-photonics-comes-to-market/, Aug. 2007.

[7] M. Paniccia et al., Integration challenge of silicon photonics with microelectronics, IEEE International Conference on Group IV Photonics, 2005. 2nd, Antwerp, Belgium, 2005, pp. 20-22.

[8] A. Liu et al., A high-speed silicon optical modulator based on a metaloxidesemiconductor capacitor, Nature volume 427, pages 615618 (12 February 2004).

[9] D. J. Thomson et al., Roadmap on silicon photonics, J. Opt. 18073003 , 2016.

[10] Yole, Silicon Photonics 2018

[11] M. A. G. Porcel et al., Monolithic photonic integration for visible and short near-infrared wavelengths: technologies and platforms for bio and life science applications, Advanced Optical Technologies, Vol. 7, no. 1-2, 2018.

[12] A. Jovic et al., A MEMS Actuator System for an Integrated 3-D Optical Coherent Tomography Scanner, Journal of Microelectromechanical Systems, vol. 27, no. 2, pp. 259-268, April 2018.

[13] Y. Li et al., Six-beam homodyne laser Doppler vibrometry based on silicon photonics, Opt. Express 26, 3638-3645 (2018).

[14] C. V. Poulton et al., Coherent solid-state LIDAR with silicon photonic optical phased arrays, Opt. Lett. 42, 4091-4094 (2017).

[15] A. Martin et al., Photonic Integrated Circuit-Based FMCW Coherent LiDAR, Journal of Lightwave Technology, vol. 36, no. 19, pp. 4640-4645, Oct.1, 2018.

[16] A. N. Tait et al., Neuromorphic photonic networks using silicon photonic weight banks, Scientific Reportsvolume 7, Article number: 7430 (2017).

[17] M. Lukosevicius et al., Reservoir Computing Approaches to Recurrent Neural Network Training, Computer Science Review, vol. 3, no. 3, pp. 127, 2009.

[18] C.-S. Poon et al., Neuromorphic silicon neurons and large-scale neural networks: challenges and opportunities. Front. Neurosci. 5, 108 (2011).

[19] N. C. Harris et al., Programmable Nanophotonics for Quantum Simulation and Machine Learning, Advanced Photonics 2017 (IPR, NOMA, Sensors, Networks, SPPCom, PS), OSA Technical Digest (online) (Optical Society of America, 2017), paper ITu3A.3.

[20] Y. Shen et al., Deep learning with coherent nanophotonic circuits, Nature Photonics volume 11, pages 441446 (2017).

[21] A. Peruzz et al., Quantum Walks of Correlated Photons, Science, 17 Sep 2010: Vol. 329, Issue 5998, pp. 1500-1503.

[22] S. Paesani et al., Experimental quantum hamiltonian learning using a silicon photonic chip and a nitrogen-vacancy electron spin in diamond, 2017 Conference on Lasers and Electro-Optics Europe \& European Quantum Electronics Conference (CLEO/Europe-EQEC), Munich, 2017, pp. 1-1.

[23] https://www.mosis.com

[24] P. Absil et al., Reliable 50Gb/s Silicon Photonics Platform for NextGeneration Data Center Optical Interconnects, $63^{\text {rd }}$ International Electronic Device Meeting, San Francisco, CA, 2017.

[25] A. E. J. Lim et al., Path to silicon photonics commercialization: $25 \mathrm{~Gb} / \mathrm{s}$ platform development in a CMOS manufacturing foundry line, OFC 2014, San Francisco, CA, 2014, pp. 1-3.

[26] B. Szelag et al., Multiple wavelength silicon photonic $200 \mathrm{~mm} R+D$ platform for $25 \mathrm{~Gb} / \mathrm{s}$ and above applications, Proceedings Volume 9891 , Silicon Photonics and Photonic Integrated Circuits V; 98911C (2016); doi: 10.1117/12.2228744, Event: SPIE Photonics Europe, 2016, Brussels, Belgium

[27] D. Knoll et al., BiCMOS silicon photonics platform for fabrication of high-bandwidth electronic-photonic integrated circuits, 2016 IEEE 16th Topical Meeting on Silicon Monolithic Integrated Circuits in RF Systems (SiRF), Austin, TX, 2016, pp. 46-49.
[28] E. Timurdogan et al., AIM Process Design Kit (AIMPDKv2.0): Silicon Photonics Passive and Active Component Libraries on a 300mm Wafer, Optical Fiber Communication Conference, OSA Technical Digest (online) (Optical Society of America, 2018), paper M3F.1.

[29] T. Aalto et al., 3 Micron Silicon Photonics, OFC 2018, San Francisco, CA, 2018, pp. 1-3.

[30] V. Stojanovi et al., Monolithic silicon-photonic platforms in state-ofthe-art CMOS SOI processes [Invited], Opt. Express 26, 13106-13121 (2018).

[31] T. Horikawa et al., A 300-mm Silicon Photonics Platform for LargeScale Device Integration, IEEE Journal of Selected Topics in Quantum Electronics, vol. 24, no. 4, pp. 1-15, July-Aug. 2018, Art no. 8200415.

[32] T. Liow et al., Silicon Modulators and Germanium Photodetectors on SOI: Monolithic Integration, Compatibility, and Performance Optimization, IEEE Journal of Selected Topics in Quantum Electronics, vol. 16, no. 1, pp. 307-315, Jan.-feb. 2010.

[33] P. Dumon, Towards Fabless Silicon Photonics, Frontiers in Optics 2008/Laser Science XXIV/Plasmonics and Metamaterials/Optical Fabrication and Testing, OSA Technical Digest (CD) (Optical Society of America, 2008), paper FMG1.

[34] M. Hochberg et al., Silicon Photonics: The Next Fabless Semiconductor Industry, in IEEE Solid-State Circuits Magazine, vol. 5, no. 1, pp. 48-58, winter 2013.

[35] J. Pond et al., A simulation tool development roadmap to support a scalable silicon photonics design ecosystem, 2015 IEEE 12th International Conference on Group IV Photonics (GFP), Vancouver, BC, 2015, pp. 189-190.

[36] M. Smit et al., An introduction to InP-based generic integration technology, Semiconductor Science and Technology, vol. 29, no. 8, Jun 13, 2014.

[37] Andy Eu-Jin Lim et al., Path to Silicon Photonics Commercialization: The Foundry Model Discussion, Silicon Photonics III Systems and Applications Editors: Pavesi, Lorenzo, Lockwood, David J (Eds.), 2016.

[38] A. E. Lim et al., Review of Silicon Photonics Foundry Efforts, IEEE Journal of Selected Topics in Quantum Electronics, vol. 20, no. 4, pp. 405-416, July-Aug. 2014, Art no. 8300112.

[39] S. J. McNab et al., Ultra-low loss photonic integrated circuit with membrane-type photonic crystal waveguides, Opt. Express 11, 2927-2939 (2003).

[40] F. Boeuf et al., Silicon Photonics R\&D and Manufacturing on 300-mm Wafer Platform, Journal of Lightwave Technology, vol. 34, no. 2, pp. 286-295, Jan.15, 152016.

[41] A. H. Atabaki et al., Integrating photonics with silicon nanoelectronics for the next generation of systems on a chip, Nature, vol. 556, 349354, (2018).

[42] T. Tsuchizawa et al., Microphotonics devices based on silicon microfabrication technology, IEEE Journal of Selected Topics in Quantum Electronics, vol. 11, no. 1, pp. 232-240, Jan.-Feb. 2005.

[43] J. S. Orcutt et. al., Open foundry platform for high-performance electronicphotonic integration, Opt. Express 20, 12222-12232 (2012).

[44] S. Assefa et al., A 90nm CMOS integrated Nano-Photonics technology for 25Gbps WDM optical communications applications, 2012 International Electron Devices Meeting, San Francisco, CA, 2012, pp. 33.8.1-33.8.3.

[45] G. Kurczveil et al., An Integrated Hybrid Silicon Multiwavelength AWG Laser, IEEE Journal of Selected Topics in Quantum Electronics, vol. 17, no. 6, pp. 1521-1527, Nov.-Dec. 2011.

[46] T. Komljenovic et al., Heterogeneous Silicon Photonic Integrated Circuits, Journal of Lightwave Technology, vol. 34, no. 1, pp. 20-35, 1 Jan.1, 2016

[47] G. Roelkens et al., III-V/silicon photonics for on-chip and intrachip optical interconnects, Laser \& Photon. Rev., 4: 751779. doi:10.1002/lpor.200900033.

[48] B. Ben Bakir et al., Electrically driven hybrid Si/III-V Fabry-Prot lasers based on adiabatic mode transformers, Opt. Express 19, 10317-10325 (2011).

[49] X. Liu et al., Mid-infrared optical parametric amplifier using silicon nanophotonic waveguides, Nature Photonics 4, 557 - 560 (2010).

[50] Milan M. Milošević et al., Rib waveguides for mid-infrared silicon photonics, J. Opt. Soc. Am. B 26, 1760-1766 (2009).

[51] M. M. Milosević et al., Silicon waveguides and devices for the midinfrared, Appl. Phys. Lett. 101, 121105 (2012)

[52] M. Muneeb et al., Demonstration of Silicon-on-insulator mid-infrared spectrometers operating at 3.8 $\mu \mathrm{m}$, Opt. Express 21, 11659-11669 (2013).

[53] K. K. Lee et al, Fabrication of ultralow-loss Si/SiO2 waveguides by roughness reduction, Opt. Lett. 26, 1888-1890 (2001).

[54] L. Chrostowski et al., Impact of fabrication non-uniformity on chipscale silicon photonic integrated circuits, Optical Fiber Communication Conference (Optical Society of America, 2014), paper Th2A.37. 
[55] F. Morichetti et al., Roughness Induced Backscattering in Optical Silicon Waveguides, Phys. Rev. Lett. 104, 033902 Published 20 January 2010.

[56] B. Peng et al., Distributed backscattering in production O-band $\mathrm{Si}$ nanophotonic waveguides, Opt. Express 25, 23477-23485 (2017).

[57] K. Okamoto, Wavelength-Division-Multiplexing Devices in Thin SOI: Advances and Prospects, IEEE Journal of Selected Topics in Quantum Electronics, vol. 20, no. 4, pp. 248-257, July-Aug. 2014, Art no. 8200410.

[58] H. Jayatilleka et al. Wavelength tuning and stabilization of microringbased filters using silicon in-resonator photoconductive heaters, Opt. Express 23, 25084-25097 (2015)

[59] P. Le Maitre et al., Impact of process variability of active ring resonators in a 300mm silicon photonic platform, 2015 European Conference on Optical Communication (ECOC), Valencia, 2015, pp. 1-3.

[60] M. Tran et al., Ultra-Low-Loss Silicon Waveguides for Heterogeneously Integrated Silicon/III-V Photonics, Appl. Sci. 2018, 8(7), 1139.

[61] F. Boeuf et al., Silicon Photonics Research and Manufacturing Using a 300-mm Wafer Platform, Silicon Photonics III Systems and Applications Editors: Pavesi, Lorenzo, Lockwood, David J (Eds.), 2016.

[62] Z. Lu et al., Performance prediction for silicon photonics integrated circuits with layout-dependent correlated manufacturing variability, Opt Express 25, 9712-9733 (2017).

[63] S. Dwivedi et al., Maximizing Fabrication and Thermal Tolerances of All-Silicon FIR Wavelength Filters, in IEEE Photonics Technology Letters, vol. 27, no. 8, pp. 871-874, April 15, 2015.

[64] Q. Lin et al., Nonlinear optical phenomena in silicon waveguides. Modeling and applications, Opt. Express 15, 16604-16644 (2007).

[65] B. Kuyken et al., Frequency conversion of mid-infrared optical signals into the telecom band using nonlinear silicon nanophotonic wires, Optical Fiber Communication Conference/National Fiber Optic Engineers Conference 2011, OSA Technical Digest (CD) (Optical Society of America, 2011), paper OThU4.

[66] B. Jalali, Silicon photonics: Nonlinear optics in the mid-infrared, Nature Photonics 4, 506 - 508 (2010)

[67] R. A. Soref et al., Large single-mode rib waveguides in GeSi-Si and Si-on-SiO2, IEEE Journal of Quantum Electronics, vol. 27, no. 8, pp. 1971-1974, Aug 1991.

[68] A. G. Rickman, G. T. Reed and F. Namavar, Silicon-on-insulator optical rib waveguide loss and mode characteristics, Journal of Lightwave Technology, vol. 12, no. 10, pp. 1771-1776, Oct. 1994.

[69] U. Fischer et al., $0.1 \mathrm{~dB} / \mathrm{cm}$ waveguide losses in single-mode SOI rib waveguides, IEEE Photonics Technology Letters, vol. 8, no. 5, pp. 647-648, May 1996.

[70] T. Aalto et al., Low-Loss Converters Between Optical Silicon Waveguides of Different Sizes and Types, Photonics Technology Letters vol. 18, No. 5, pp. 709-711, 2006.

[71] S. Nakamura et al., Wavelength selective switching with one-chip silicon photonic circuit including $8 \times 8$ matrix switch, 2011 Optical Fiber Communication Conference and Exposition and the National Fiber Optic Engineers Conference, Los Angeles, CA, 2011, pp. 1-3.

[72] B. Schueppert et al., Integrated optics in Si and SiGe-heterostructures, vol. 14, no. 10, pp. 2311-2323, Oct. 1996.

[73] T. Aalto et al., Launching of multi-project wafer runs in ePIXfab with micron-scale silicon rib waveguide technology, Proceedings of SPIE, vol. 8990 (Photonics West, San Francisco, USA, 3-5 February, 2014), Paper 899003, 2014.

[74] L. Zimmermann et al., C-Band Optical 90-Hybrids Based on Siliconon-Insulator $4 \times 4$ Waveguide Couplers, IEEE Photonics Tech. Letters, vol. 21, nr. 3, 2009.

[75] S. A. Miller et al., Low-loss silicon platform for broadband mid-infrared photonics, Optica 4, 707-712 (2017).

[76] Y. Z. Tang et al., Integrated waveguide turning mirror in silicon-oninsulator, IEEE Photonics Technology Letters, vol. 14, no. 1, pp. 68-70, Jan. 2002.

[77] M. Cherchi et al., Dramatic size reduction of waveguide bends on a micron-scale silicon photonic platform, Opt. Express 21, 17814-17823 (2013).

[78] K. Vyrsokinos et al., DPSK-Demodulation based on Ultra-Compact micron-scale SOI platform, Optical Fiber Communication Conference, OSA Technical Digest (online) (Optical Society of America, 2015), paper W2A.14.

[79] M. Pagani et al., Low-error and broadband microwave frequency measurement in a silicon chip, Optica 2, 751-756 (2015).

[80] M. Moralis-Pegios et al., Multicast-Enabling Optical Switch Design Employing Si Buffering and Routing Elements, IEEE Photonics Technology Letters 30 (8), April 15, pp. 712715, 2018.
[81] M. Harjanne et al., Sub- $\mu$ s switching time in silicon-on-insulator Mach Zehnder thermo-optic switch, Photonics Technology Letters, vol. 16, No 9, pp. 2039-2041, 2004.

[82] D. Feng et al., High-Speed GeSi Electroabsorption Modulator on the SOI Waveguide Platform, IEEE Journal of Selected Topics in Quantum Electronics, vol. 19, no. 6, pp. 64-73, Nov.-Dec. 2013, Art no. 3401710

[83] D. Feng et al., High-Speed Receiver Technology on the SOI Platform, IEEE Journal of Selected Topics in Quantum Electronics, vol. 19, no. 2, Mar.-Apr. 2013, Art no. 3800108. 2004.

[84] ] C. Minkenberg et al., Reimagining Datacenter Topologies With Integrated Silicon Photonics, IEEE/OSA Journal of Optical Communications and Networking, vol. 10, no. 7, pp. 126-139, July 2018.

[85] C. Gunn, CMOS photonics for high speed interconnects, IEEE Micro 26(2), 5866 (2006).

[86] G. Denoyer et al., Hybrid Silicon Photonic Circuits and Transceiver for $50 \mathrm{~Gb} / \mathrm{s}$ NRZ Transmission Over Single-Mode Fiber, Journal of Lightwave Technology, vol. 33, no. 6, pp. 1247-1254, March15, 152015.

[87] X. R. Zhang et al., Copper pillar bump structure optimization for flip chip packaging with Cu/Low-K stack, 2010 11th International Thermal, Mechanical \& Multi-Physics Simulation, and Experiments in Microelectronics and Microsystems (EuroSimE), Bordeaux, 2010, pp. 1-7.

[88] J. M. Fedeli et al., PhotonicElectronic Integration With Bonding, IEEE Journal of Selected Topics in Quantum Electronics, vol. 20, no. 4, pp. 350-358, July-Aug. 2014.

[89] Y. Chen et al., A 25Gb/s hybrid integrated silicon photonic transceiver in 28nm CMOS and SOI, 2015 IEEE International Solid-State Circuits Conference - (ISSCC) Digest of Technical Papers, San Francisco, CA, 2015, pp. 1-3.

[90] P. De Dobbelaere et al.Advanced silicon photonics technology platform leveraging a semiconductor supply chain, 2017 IEEE International Electron Devices Meeting (IEDM), San Francisco, CA, 2017, pp. 34.1.1-34.1.4.

[91] C. Battenet al., Building Many-Core Processor-to-DRAM Networks with Monolithic CMOS Silicon Photonics, IEEE Micro, vol. 29, no. 4, pp. 8-21, July/Aug. 2009.

[92] D. J. Shin et al., Integration of silicon photonics into DRAM process, 2013 Optical Fiber Communication Conference and Exposition and the National Fiber Optic Engineers Conference (OFC/NFOEC), Anaheim, CA, 2013, pp. 1-3.

[93] M. Georgas et al., A monolithically-integrated optical transmitter and receiver in a zero-change 45nm SOI process, 2014 Symposium on VLSI Circuits Digest of Technical Papers, Honolulu, HI, 2014, pp. 1-2.

[94] S. Assefa et al. A 90nm CMOS integrated nano-photonics technology for 25Gbps WDM optical communications applications, Proceedings of the International Electron Devices Meeting (2012) pp. 33.8.133.8.3.

[95] H. Abediasl and Hossein Hashemi, Monolithic optical phased-array transceiver in a standard SOI CMOS process, Opt. Express 23, 6509-6519 (2015).

[96] L. Zimmermann et al., Monolithically integrated 10Gbit/sec Silicon modulator with driver in $0.25 \mu \mathrm{m}$ SiGe:C BiCMOS, 39th European Conference and Exhibition on Optical Communication (ECOC 2013), London, 2013, pp. 1-3.

[97] J. S. Orcutt et al., Nanophotonic integration in state-of-the-art CMOS foundries, Opt. Express 19, 2335-2346 (2011).

[98] D. Knoll et al., Monolithically Integrated 25Gbit/sec Receiver for $1.55 \mu \mathrm{m}$ in Photonic BiCMOS Technology, Optical Fiber Communications (OFC) Conference, Th4C.4, 2014

[99] C. H. Henry et al., Low loss Si3N4SiO2 optical waveguides on Si, Appl. Opt. 26, 2621-2624 (1987).

[100] J. F. Bauters et al., Ultra-low-loss Single-mode Si3N4 Waveguides with $0.7 \mathrm{~dB} / \mathrm{m}$ Propagation Loss, 37th European Conference and Exposition on Optical Communications, OSA Technical Digest (CD) (Optical Society of America, 2011), paper Th.12.LeSaleve.3.

[101] A. Rahim et al., Expanding the Silicon Photonics Portfolio With Silicon Nitride Photonic Integrated Circuits, Journal of Lightwave Technology, vol. 35, no. 4, pp. 639-649, Feb.15, 152017.

[102] M. J. R. Heck et al, Ultra-low loss waveguide platform and its integration with silicon photonics, Laser and Photonics Reviews, Volume 8, Issue 5, September 2014, Pages 667686.

[103] C. G. H. Roeloffzen et al., Low-Loss Si3N4 TriPleX Optical Waveguides:Technology and Applications Overview, IEEE J. of Selected Topics in Quantum Electronics, vol. 24, no. 4, Jul/Aug. 2018.

[104] A. Leinse et al., TriPleX: The versatile silicon nitride waveguide platform, 2016 Progress in Electromagnetic Research Symposium (PIERS), Shanghai, 2016, pp. 67-67.

[105] L. Hoffman et al., Low Loss CMOS-Compatible PECVD Silicon Nitride Waveguides and Grating Couplers for Blue Light Optogenetic Applications, IEEE Photonics Journal, vol. 8, no. 5, pp. 1-11, Oct. 2016, Art no. 2701211 
[106] Z. Zhang et al., A silicon nitride platform by physical vapor deposition for dense wavelength division multiplexing on chip, 11th International Conference on Group IV Photonics (GFP), Paris, 2014, pp. 193-194.

[107] C. Baudot et al., Advanced solutions in silicon photonics using traditional fabrication methods and materials of CMOS technologies, Proceedings Volume 10537, Silicon Photonics XIII; 105370G (2018).

[108] S. Guerber et al., Integrated SiN on SOI dual photonic devices for advanced datacom solutions, Proceedings Volume 10686, Silicon Photonics: From Fundamental Research to Manufacturing; 106860W (2018) https://doi.org/10.1117/12.2306160.

[109] J. F. Song et al., Silicon Nitride-Based Compact Double-Ring Resonator Comb Filter With Flat-Top Response, IEEE Photonics Technology Letters, vol. 20, no. 24, pp. 2156-2158, Dec.15, 2008.

[110] Y. Huang et al ., CMOS compatible monolithic multi-layer Si3N4on-SOI platform for low-loss high performance silicon photonics dense integration, Opt. Express 22, 21859-21865 (2014).

[111] D. J. Moss et al., New CMOS-compatible platforms based on silicon nitride and Hydex for nonlinear optics, Nature Photon., vol. 7, pp. 597607, 2013. doi: 10.1038 /nphoton.2013.183

[112] J. F. Bauters et al., Ultra-low-loss high-aspect-ratio Si3N4 waveguides, Opt. Express, vol. 19, pp. 31633174, 2011.

[113] D. Melati et al., Real photonic waveguides: guiding light through imperfections, Adv. Opt. Photon. 6, 156-224 (2014).

[114] M. H. P. Pfeiffer et al., Photonic Damascene process for integrated high-Q microresonator based nonlinear photonics, Optica 3, $20-25$ (2016).

[115] B. Kuyken, Four-Wave-Mixing in Dispersion-Engineered Silicon Nanophotonic Circuits for Telecommunication and Sensing Applications, $\mathrm{Ph} . \mathrm{D}$ Thesis, Ghent University (Belgium), 2012-2013.

[116] J. S. Penades et al., Low-Loss Mid-Infrared SOI Slot Waveguides, IEEE Photonics Technology Letters, vol. 27, no. 11, pp. 1197-1199, March 24 2015.

[117] T. Hu et al., Silicon photonic platforms for mid-infrared applications [Invited], Photon. Res. 5, 417-430 (2017).

[118] J. S. Penades et al. Suspended silicon mid-infrared waveguide devices with subwavelength grating metamaterial cladding, Opt. Express 24, 22908-22916 (2016)

[119] J. S. Penades et al, Suspended silicon waveguides for long-wave infrared wavelengths, Opt. Lett. 43, 795-798 (2018).

[120] W. Zhou et al., Fully suspended slot waveguides for high refractive index sensitivity, Optics Letters 42, 1245 (2017).

[121] W. Zhou et al., Fully suspended slot waveguide platform, Journal of Applied Physics 123, 063103 (2018).

[122] R. Soref, Mid-infrared photonics in silicon and germanium, Nature Photon., vol. 4, pp. 495497, 2010.

[123] A. Malik et al., Germanium-on-silicon mid-infrared waveguides and Mach-Zehnder interferometers, IEEE Photonics Conference, 2013 pp. 103104.

[124] A. Malik et al., Ge-on-Si wavelength division multiplexing components near $4.7 \mu \mathrm{m}$, Conference on Lasers and Electro-Optics, OSA Technical Digest (online) (Optical Society of America, 2018), paper JW2A.39.

[125] J. Fedeli and S. Nicoletti, Mid-Infrared (Mid-IR) Silicon-Based Photonics, Proceedings of the IEEE.

[126] V. M. Lavchiev and B. Jakoby, Photonics in the Mid-Infrared: Challenges in Single-Chip Integration and Absorption Sensing, IEEE Journal of Selected Topics in Quantum Electronics, vol. 23, no. 2, pp. 452-463, March-April 2017.

[127] U. Younis et al., Germanium-on-SOI waveguides for mid-infrared wavelengths, Opt. Express 24, 11987-11993 (2016).

[128] U. Younis et al., Towards low-loss waveguides in SOI and Ge-on-SOI for mid-IR sensing, Journal of Physics Communications, Volume 2, Number 4.

[129] J. Kang et al., Novel Ge waveguide platform on Ge-on-insulator wafer for mid-infrared photonic integrated circuits, Opt. Express 24, 1185511864 (2016).

[130] W. Li et al., Germanium-on-silicon nitride waveguides for mid-infrared integrated photonics, Appl. Phys. Lett. 109, 241101 (2016).

[131] P. Barritault et al., Design, fabrication and characterization of an AWG at 4.5 $\mu \mathrm{m}$, Opt. Express 23, 26168-26181 (2015)

[132] J. Kang et al., Focusing subwavelength grating coupler for mid-infrared suspended membrane germanium waveguides, Optics Letters 42, 2094 (2017)

[133] T.-H. Xiao et al., Mid-infrared high-Q germanium nanocavity, Photonics Research 6, 925 (2018).

[134] D. X. Xu et al., Silicon Photonic Integration PlatformHave We Found the Sweet Spot?, IEEE Journal of Selected Topics in Quantum Electronics, vol. 20, no. 4, pp. 189-205, July-Aug. 2014.
[135] J. K. S. Poon et al., Integrated photonic devices and circuits in multilayer silicon nitride-on-silicon platforms, Proc. Opt. Fiber Commun. Conf. Exhib., Los Angeles, CA, 2015, pp. 13. doi: 10.1364/OFC. 2015.Th3F.1.

[136] J. F. Bauters et al., Silicon on ultra-low-loss waveguide photonic integration platform, Opt. Express 21, 544-555 (2013).

[137] W. D. Sacher et al., Tri-layer silicon nitride-on-silicon photonic platform for ultra-low-loss crossings and interlayer transitions, Opt. Express 25 , 30862-30875 (2017)

[138] Y. Huang et al., CMOS compatible monolithic multi-layer Si3N4-on-SOI platform for low-loss high performance silicon photonics dense integration, Opt. Express 22, 21859-21865 (2014).

[139] W. D. Sacher et al.,Monolithically Integrated Multilayer Silicon Nitrideon-Silicon Waveguide Platforms for 3-D Photonic Circuits and Devices, in Proceedings of the IEEE. doi: 10.1109/JPROC.2018.2860994

[140] C. Doerr et al., Single-chip silicon photonics 100-Gb/s coherent transceiver, Proc. Opt. Fiber Commun. Conf. Exhib., San Francisco, CA, 2014, pp. 13.

[141] L. Chen et al., Monolithic silicon chip with 10 modulator channels at 25 Gbps and 100-GHz spacing, Opt. Express, vol. 19, pp. B946B951, 2011.

[142] R. Baets, Building a sustainable future for silicon photonics, 8th International Conference on Group IV Photonics 2011, United Kingdom, p. 3-4 (PLE2) (2011).

[143] A. Novack et al., Progress in silicon platforms for integrated optics, Nanophotonics, 3(4-5), pp. 205-214. Retrieved 18 Sep. 2018, from doi:10.1515/nanoph-2013-0034.

[144] http://ai.eecs.umich.edu/people/conway/Impact/FundingaRevolution. html

[145] M. Hochberg \& T. Baehr-Jones, Towards fabless silicon photonics, Nature Photonics, 10.1038/nphoton.2010.172.

[146] R. Baets et al., Silicon Photonics, 2007 International Symposium on VLSI Technology, Systems and Applications (VLSI-TSA), Hsinchu, 2007, pp. 1-3.

[147] F. Boeuf et al., Recent progress in Silicon Photonics $R \& D$ and manufacturing on 300mm wafer platform, 2015 Optical Fiber Communications Conference and Exhibition (OFC), Los Angeles, CA, 2015, pp. 1-3.

[148] http://www.europractice-ic.com

[149] M. Liehr et al., AIM Photonics - What merging photonics with nanoelectronics will do, 2016 IEEE Optical Interconnects Conference (OI), San Diego, CA, 2016, pp. 22-23.

[150] T. L. Koch et al., The American Institute for Manufacturing Integrated Photonics: advancing the ecosystem, Proc. SPIE 9772, Broadband Access Communication Technologies X, 977202 (12 February 2016).

[151] M. Heins et al., Design Flow Automation for Silicon Photonics. Challenges, Collaboration, and Standardization, Silicon Photonics III Systems and Applications Editors: Pavesi, Lorenzo, Lockwood, David J (Eds.), 2016

[152] W. Bogaerts, L. Chrostowski, Silicon Photonics Circuit Design: Methods, Tools and Challenges, Laser \& Photonics Review, Mar. 2018.

[153] Z. Zhang et al., Compact Modeling for Silicon Photonic Heterogeneously Integrated Circuits, Journal of Lightwave Technology, vol. 35, no. 14, pp. 2973-2980, July15, 152017.

[154] L. Chrostowski \& M. Hochberg, Silicon Photonics Design From Devices to Systems, Cambridge University Press 978-1-107-08545-9.

[155] X. Chen et al., The emergence of silicon photonics as a flexible technology platform, Proceedings of the IEEE, 2018 (invited).

[156] T. Mogami et al., High-performance silicon photonics process platform for low-power photonic integrated circuits, 2016 IEEE Silicon Nanoelectronics Workshop (SNW), Honolulu, HI, 2016, pp. 216-217.

[157] S. K. Selvaraja et al., Highly uniform and low-loss passive silicon photonics devices using a $300 \mathrm{~mm}$ CMOS platform, Proc. Opt Fiber Commun. Conf. Exhib., San Francisco, CA, 2014, pp. 13. doi: 10.1364/OFC.2014.Th2A.33

[158] J. Park et al., Performance improvement in silicon arrayed waveguide grating by suppression of scattering near the boundary of a star coupler, Appl. Opt., vol. 54, no. 17, pp. 55975602, Jun. 2015

[159] T. Horikawa et al., The impacts of fabrication error in Si wirewaveguides on spectral variation of coupled resonator optical waveguides, Microelectron. Eng., vol. 156, pp. 4649, Apr. 2016.

[160] T. Horikawa et al.,Resonant wavelength variation modelling for microring resonators based on fabrication deviation analysis, Proc. 43rd Eur. Conf. Opt. Commun., Gothenburg, Sweden, Sep. 2017.

[161] H. Okayama et al., Low loss $100 \mathrm{GHz}$ spacing Si arrayed-waveguide grating using minimal terrace at slab-array interface, Electron. Lett., vol. 52 , no. 18 , pp. 15451546 , Sep. 2016.

[162] Miaofeng Li et al., Silicon intensity MachZehnder modulator for single lane $100 \mathrm{~Gb} / \mathrm{s}$ applications, Photon. Res. 6, 109-116 (2018). 
[163] X. Xiao et al., High Speed Silicon Photonic Modulators, Optical Fiber Communication Conference, OSA Technical Digest (online) (Optical Society of America, 2017), paper Tu2H.1.

[164] E. Timurdogan et al., An ultralow power athermal silicon modulator, Nature Communications volume 5, Article number: 4008 (2014).

[165] K. Goi et al., DQPSK/QPSK Modulation at 40-60 Gb/s using Low-Loss Nested Silicon Mach-Zehnder Modulator, Optical Fiber Communication Conference/National Fiber Optic Engineers Conference 2013, OSA Technical Digest (online) (Optical Society of America, 2013), paper OW4J.4.

[166] B. Milivojevic et al., Demonstration of Optical Transmission at Bit Rates of up to 321.4Gb/s using Compact Silicon Based Modulator and Linear BiCMOS MZM Driver, Optical Fiber Communication Conference, OSA Technical Digest (online) (Optical Society of America, 2016), paper Th1F.2.

[167] A. Samani et al., A Low-Voltage 35-GHz Silicon Photonic ModulatorEnabled 112-Gb/s Transmission System, IEEE Photonics Journal, vol. 7, no. 3, pp. 1-13, June 2015

[168] W. D. Sacher et al., Wide bandwidth and high coupling efficiency $\mathrm{Si}_{3} \mathrm{~N}_{4}$-on-SOI dual-level grating coupler, Opt. Express 22, 10938-10947 (2014).

[169] J. Notaros et al., Ultra-efficient CMOS fiber-to-chip grating couplers, 2016 Optical Fiber Communications Conference and Exhibition (OFC), Anaheim, CA, 2016, pp. 1-3.

[170] W. Zhou et al., Tailorable dual-wavelength-band coupling in a transverse-electric-mode focusing subwavelength grating coupler, Opt. Lett. 43, 2985-2988 (2018).

[171] T. Barwicz and Y. Taira, Low-cost interfacing of fibers to nanophotonic waveguides: Design for fabrication and assembly tolerances, IEEE Photonics J., vol. 6, no. 4, 2014

[172] C. E. Png et al., Optimized optical devices for edge-coupling-enabled silicon photonics platform, Proceedings Volume 10537, Silicon Photonics XIII; 105371L (2018).

[173] R. Marchetti et al., High-efficiency grating-couplers: demonstration of a new design strategy, Scientific Reportsvolume 7, Article number: 16670 (2017).

[174] F. Eltes et al., A novel 25 Gbps electro-optic Pockels modulator integrated on an advanced Si photonic platform, 2017 IEEE International Electron Devices Meeting, December 2-6, 2017.

[175] K. Alexander et al., Broadband Electro-optic Modulation using Low loss PZT-on-Silicon Nitride Integrated Waveguides, Conference on Lasers and Electro-Optics, OSA Technical Digest (online) (Optical Society of America, 2017), paper JTh5C.7.

[176] B. Ben Bakir et al., Low-Loss $(<1 d B)$ and Polarization-Insensitive Edge Fiber Couplers Fabricated on 200-mm Silicon-on-Insulator Wafers, IEEE Photonics Technology Letters, vol. 22, no. 11, pp. 739-741, June1, 2010.

[177] G. H. Duan et al., Hybrid III-V on Silicon Lasers for Photonic Integrated Circuits on Silicon, IEEE Journal of Selected Topics in Quantum Electronics, vol. 20, no. 4, pp. 158-170, July-Aug. 2014

[178] L. Diang and J. E. Bowers, Recent progress in lasers on silicon, Nature Photonics volume 4, pages 511517 (2010).

[179] T. Shimizu et al., High density hybrid integrated light source with a laser diode array on a silicon optical waveguide platform for inter-chip optical interconnection, 8th IEEE International Conference on Group IV Photonics, London, 2011, pp. 181-183.

[180] J. B. Rodriguez et al., Room-temperature operation of a $2.25 \mu \mathrm{m}$ electrically pumped laser fabricated on a silicon substrate, Appl. Phys. Lett. 94, 061124 (2009).

[181] Z. Whang et al., Room-temperature InP distributed feedback laser array directly grown on silicon, Nature Photonics volume 9, pages 837842 (2015).

[182] S. Chen et al., Electrically pumped continuous-wave IIIV quantum dot lasers on silicon, Nature Photonics volume 10, pages 307311 (2016).

[183] s. Wirths et al., Lasing in direct-bandgap GeSn alloy grown on Si, Nature Photonics volume 9, pages 8892 (2015).

[184] Y. Urino et al., First Demonstration of Athermal Silicon Optical Interposers With Quantum Dot Lasers Operating up to $125{ }^{\circ} \mathrm{C}$, Journal of Lightwave Technology, vol. 33, no. 6, pp. 1223-1229, 15 March15, 2015.

[185] T. Wang et al., 1.3- $\mu \mathrm{m}$ InAs/GaAs quantum-dot lasers monolithically grown on Si substrates, Opt. Express 19, 11381-11386 (2011).

[186] S. Chen et al., 1.3- $\mu \mathrm{m}$ InAs/GaAs quantum-dot laser monolithically grown on Si substrates operating over 100C, Electron. Lett. 50, 14671468 (2014).

[187] G. Roelkens et al., III-V-on-Silicon Photonic Devices for Optical Communication and Sensing, Photonics 2015, 2(3), 969-1004.
[188] A Y. Liu et al., Quantum dot lasers for silicon photonics [Invited], Photon. Res. 3, B1-B9 (2015).

[189] K. Tanabe and Y. Arakawa, 1.3 m InAs/GaAs Quantum Dot Lasers on SOI Waveguide Structures, CLEO: 2014, OSA Technical Digest (online) (Optical Society of America, 2014), paper STh1G.6.

[190] B. Szelag et al., Hybrid III-V/Si DFB laser integration on a $220 \mathrm{~mm}$ fully CMOS-compatible silionn photonlcsplotform, 2017 IEEE International Electron Devices Meeting (IEDM), San Francisco, CA, 2017, pp. 24.1.124.1.4.

[191] J. Zhang et al., Transfer-printing-based integration of a III-V-on-silicon distributed feedback laser, Opt. Express 26, 8821-8830 (2018).

[192] T. Horikawa et al., Process control and monitoring in device fabrication for optical interconnection using silicon photonics technology, 2015 IEEE International Interconnect Technology Conference and 2015 IEEE Materials for Advanced Metallization Conference (IITC/MAM), Grenoble, 2015, pp. 277-280.

[193] F. Boeuf et al., Silicon Photonics $R \& D$ and Manufacturing on 300-mm Wafer Platform, Journal of Lightwave Technology, vol. 34, no. 2, pp. 286-295, 15 Jan.15, 2016.

[194] W. A. Zortman et al., Silicon photonics manufacturing, Opt. Express 18, 23598-23607 (2010).

[195] J. De Coster et al., Test-station for flexible semi-automatic wafer-level silicon photonics testing,Proc. IEEE Eur. Test Symp., Amsterdam, The Netherlands, May 2016.

[196] C. Sorace-Agaskar et al., Electro-optical co-simulation for integrated CMOS photonic circuits with VerilogA, Opt. Express 23, 27180-27203 (2015).

[197] The PDAFlow foundation, http://www.pdaflow.org.

[198] A. Farsaei et al., A Novel and Scalable Design Methodology for the Simulation of Photonic Integrated Circuits, Advanced Photonics 2016 (IPR, NOMA, Sensors, Networks, SPPCom, SOF), OSA Technical Digest (online) (Optical Society of America, 2016), paper JTu4A.2.

[199] W. Bogaerts et al., The IPKISS photonic design framework, Optical Fiber Communication Conference, OSA Technical Digest (online) (Optical Society of America, 2016), paper W1E.1.

[200] J. Pond et al., Design and optimization of photolithography friendly photonic components, SPIE OPTO Proceedings Volume 9751, Smart Photonic and Optoelectronic Integrated Circuits XVIII; 97510V (2016).

[201] D. Celo et al., Optical proximity correction in geometry sensitive silicon photonics waveguide crossings, 2017 IEEE 14th International Conference on Group IV Photonics (GFP), Berlin, 2017, pp. 45-46.

[202] W. Bogaerts, M. Fiers and P. Dumon, Design Challenges in Silicon Photonics, IEEE Journal of Selected Topics in Quantum Electronics, vol 20, no. 4, pp. 1-8, July-Aug. 2014.

[203] N. Pavarelli et al., Optical and electronic packaging process for silicon photonic systems, 2014 The European Conference on Optical Communication (ECOC), Cannes, 2014, pp. 1-4.

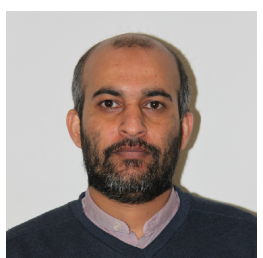

Abdul Rahim is currently a post-doc with Professor Roel Baets at the photonics research group of Ghent University. Apart from his involvement in silicon photonics design and characterization activities at Photonics Research Group, he is managing the activities of ePIXfab, the European Silicon Photonics Alliance. From 2009 to 2014 he worked with the research group of Professor Klaus Petermann and was awarded a doctorate degree by Technische Universitaet Berlin for his work on investigating novel silicon photonic integrated filters for optical

communication systems. 


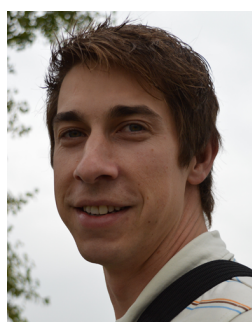

Thijs Spuesens received (S08M08) received the M.Sc. degree in electrical engineering from the Eindhoven University of Technology, Eindhoven, The Netherlands, in 2008. In 2008 he joined the Photonics Research Group of Ghent Universityimec, where he received the Ph.D. degree in photonics in 2014 During his Ph.D. he was involved in the FP7 projects WADIMOS and HISTORIC, where he worked on the integration of III-V microsources and detectors on silicon for on-chip optical interconnects. He was involved in the FP7 project Plat4M, where he has worked on platform development for silicon photonics.

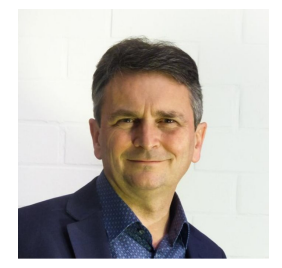

Roel Baets is full professor at Ghent University (UGent) and is part-time associated with IMEC. He received an MSc degree in Electrical Engineering from UGent in 1980 and a second MSc degree from Stanford in 1981. He received a PhD degree from UGent in 1984. From 1984 till 1989 he held a postdoctoral position at IMEC. Since 1989 he has been a professor in the Faculty of Engineering and Architecture of UGent where he founded the Photonics Research Group. From 1990 till 1994 he has also been a part-time professor at Delft University of Technology and from 2004 till 2008 at Eindhoven University of Technology. Roel Baets has mainly worked in the field of integrated photonics. He has made contributions to research on photonic integrated circuits, both in III-V semiconductors and in silicon, as well as their applications in telecom, datacom, sensing and medicine. Web of Science reports over 600 publications with an h-index well over 60 . As part of a team of 8 professors Roel Baets leads the Photonics Research Group. With about 90 researchers this group is involved in numerous (inter)national research programs and has created six spin-off companies. The silicon photonics activities of the group are part of a joint research initiative with IMEC. Roel Baets has led major research projects in silicon photonics in Europe. In 2006 he founded ePIXfab, the globally first Multi-Project-Wafer service for silicon photonics. Since then ePIXfab has evolved to become the European Silicon Photonics Alliance. Roel Baets is also director of the multidisciplinary Center for Nano- and Biophotonics (NB Photonics) at UGent, founded in 2010. He was co-founder of the European MSc programme in Photonics. Roel Baets is an ERC grantee of the European Research Council and a Methusalem grantee of the Flemish government. He is a Fellow of the IEEE, of the European Optical Society (EOS) and of the Optical Society (OSA). He is also a member of the Royal Flemish Academy of Belgium for Sciences and the Arts. He has been a recipient of the 2011 MOC award and of the 2018 PIC-International Lifetime Achievement Award. He is Director-at-Large in the Board of Directors of The Optical Society (OSA).

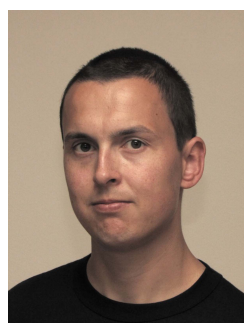

Wim Bogaerts is professor in the Photonics Research Group at Ghent University - imec. He received his $\mathrm{PhD}$ in the modelling, design and fabrication of silicon nanophotonic components at Ghent University in 2004. During this work, he started the first silicon photonics process on imec's $200 \mathrm{~mm}$ pilot line, which formed the basis of the multi-project-wafer service ePIXfab. Wim's current research focuses on the challenges for large-scale silicon photonics: Design methodologies and controllability of complex photonic circuits.

In 2014, Wim co-founded Luceda Photonics, a spin-off company of Ghent University, IMEC and the University of Brussels (VUB). Luceda Photonics develops unique software solutions for silicon photonics design, using the IPKISS design framework.

Since 2016 Wim is again full-time professor at Ghent University, looking into novel topologies for large-scale programmable photonic circuits, supported by a consolidator grant of the European Research Council (ERC).

Wim has a strong interest in telecommunications, information technology and applied sciences. He is a member of IEEE, Optical Society of America (OSA) and SPIE. 